\title{
Flight Test of Composite Model Reference Adaptive Control (CMRAC) Augmentation Using NASA AirSTAR Infrastructure
}

\author{
Irene M. Gregory ${ }^{1}$ \\ NASA Langley Research Center, Hampton, VA 23681 \\ Ross Gadient ${ }^{2}$ and Eugene Lavretsky ${ }^{3}$ \\ The Boeing Company, Huntington Beach, California 92647
}

\begin{abstract}
This paper presents flight test results of a robust linear baseline controller with and without composite adaptive control augmentation. The flight testing was conducted using the NASA Generic Transport Model as part of the Airborne Subscale Transport Aircraft Research system at NASA Langley Research Center.
\end{abstract}

\section{Introduction}

This paper is devoted to the presentation of subscale flight test results collected as part of the Integrated Resilient Aircraft Control (IRAC) project under the NASA Aviation Safety Program. The use of the Airborne Subscale Transport Aircraft Research (AirSTAR) [1]-[6] facility at NASA Langley Research Center allows for the rapid prototyping and testing of control algorithms in high fidelity simulation and flight testing environments. Specifically, this paper presents the results of flight testing that employed the Generic Transport Model (GTM), which is a dynamically scaled turbine-powered aircraft. The flight test results presented here compare robust linear baseline control with and without Combined/Composite Model Reference Adaptive Control (CMRAC) architecture. The CMRAC architecture is an extension of the now-classical Model Reference Adaptive Control (MRAC) [7], [8], [9], with detailed theoretical development of CMRAC available in [10]. Details on the design models and specific control law parameters used in the flight testing with corresponding simulation analyses may be found in [11].

The rest of the paper is organized as follows. In Section II, an overview of the AirSTAR system and flight test implementation details are included. In Section III, flight test results from piloted evaluations performed in September 2010 are presented. Finally, conclusions are presented in Section IV.

\section{AirSTAR Overview and Flight Control System Application}

The Airborne Subscale Transport Aircraft Research (AirSTAR) facility at the NASA Langley Research Center has been designed to provide a flexible research environment with the ability to conduct rapid prototyping and testing of control algorithms in extremely adverse flight conditions. AirSTAR is an integrated flight test infrastructure which currently utilizes a number of remotely piloted aircraft for flight testing. One particular use of AirSTAR is flight testing research control laws in adverse flight conditions. During this set of flight tests, AirSTAR consisted of a remotely piloted dynamically scaled turbine-powered test aircraft, the Mobile Operations Station (MOS) (an integrated ground station and control room), and a test range. Under the current AirSTAR Concept of Operations (CONOPS) (Figure II.1) a safety pilot, using a hobbyist radio control transmitter, performs the takeoff and climbs to a specified altitude, where control of the aircraft is transferred to a research pilot through a handoff maneuver. The research pilot executes the flight test plan from a research cockpit located in the MOS, which utilizes synthetic vision displays driven with aircraft sensor data. The research pilot uses a ground-based flight control system (FCS) that is connected to the aircraft through an L-band telemetry uplink and S-band telemetry

\footnotetext{
${ }^{1}$ Senior Research Engineer, Dynamic Systems and Control Branch, AIAA Assoc. Fellow.

${ }^{2}$ Engineer, The Boeing Company, AIAA Senior Member.

${ }^{3}$ Sr. Technical Fellow, The Boeing Company, AIAA Assoc. Fellow, IEEE Sr. Member.
} 
downlink. Once the flight test maneuvers are complete, the safety pilot resumes control of the aircraft and performs the landing. The safety pilot is the pilot-in-command of the aircraft and determines who is in control of the aircraft (physically and procedurally) at all times via a switch on the safety pilot's transmitter. When the onboard flight control unit (FCU) receives the appropriate command from the safety pilot, the FCU begins responding to the research pilot's commands received through the L-band telemetry uplink. The command state of the FCU (i.e. who actually has control of the aircraft) is part of the data on the S-band telemetry downlink.

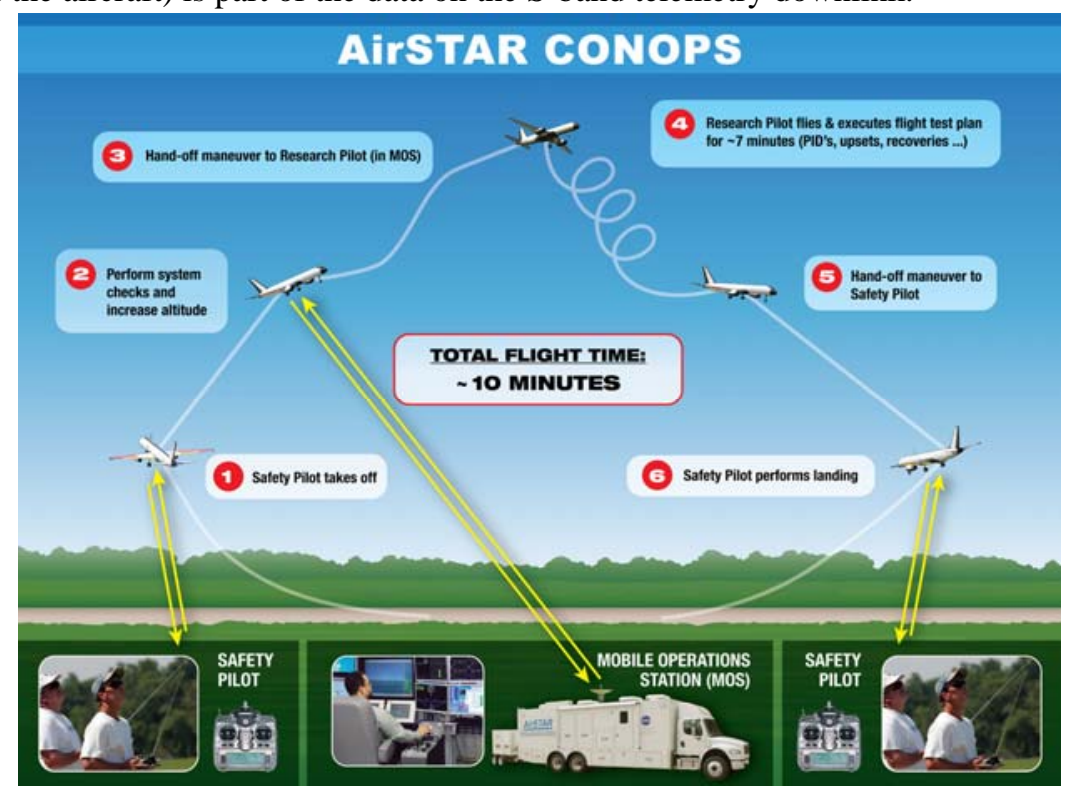

Figure II.1. AirSTAR Concept of Operations

For the purposes of this work, AirSTAR operates a fully instrumented test aircraft (Figure II.2). The test aircraft is a 5.5\% dynamically scaled twin turbine-powered generic transport model (GTM). Dynamic scaling (i.e., similitude using equal Froude number and relative density between model-scale and full-scale) allows subscale flight test results to be applied to full-scale aircraft. This model (tail number T2) has a $6.5 \mathrm{ft}$ wingspan, weighs 54 lbs at takeoff, and has a flight time of approximately 15 minutes. The aircraft is outfitted with full flight test instrumentation, including angle of attack and angle of sideslip vanes, static and dynamic pressure, control surface positions, rate gyros, accelerometers, a 6-DOF INS/GPS package, and engine instrumentation. Downlink data update rates vary from $5 \mathrm{~Hz}$ on the GPS data to $200 \mathrm{~Hz}$ on the data from analog sensors. Uplink commands are transmitted at $200 \mathrm{~Hz}$.

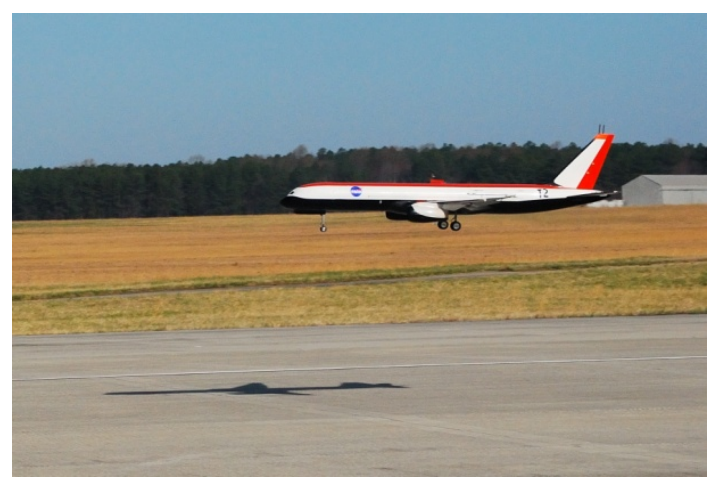

Figure II.2. AirSTAR Test Aircraft T2

The vehicle and operational concept used by AirSTAR present some unique challenges: First and foremost, dynamically scaling an aircraft results in increased model airspeed, angular rates, wing loading, and increased pilot workload relative to a model that is only geometrically scaled. These factors make a dynamically scaled model more challenging to fly and less forgiving of mistakes. Second, under the current AirSTAR CONOPS, the aircraft must remain within visual range of the safety pilot, effectively limiting the test volume to an approximately $1 / 2$-mile radius 
circle around the safety pilot, extending to $\sim 2000 \mathrm{ft}$ above the surface in altitude. Limited test volume combined with the relatively high airspeeds of the test aircraft adds to the pilot's workload. Third, one of the primary purposes of AirSTAR is to perform flight research and flight validation of multiple research control laws in adverse flight conditions, which presents unique challenges to flight testing in an efficient and safe manner.

The AirSTAR FCS uses a reversionary build-up approach to mitigate the risks associated with flight testing complex research control laws. Complexity is added in distinct stages that can be quickly transitioned using a twoswitch "arm" and "engage" process. The FCS is separated into three flight control law (FCL) modes, shown in Figure II.3. These three modes are mutually exclusive; only one can be active at a time. Mode 1 is a stick-to-surface control law composed of stick shaping only; no sensor feedback is used. This mode is the reversionary control law and is simple by design. Mode 2 is the original in-house baseline FCL, containing a conventional (non-adaptive) closed-loop controller. Mode 3 is reserved for the research control laws, and can contain any number of FCLs, although only one can be operational at any given time. To further enhance safety, Mode 3 FCLs reside on a separate processor from the rest of the flight software.

The FCS also contains three auxiliary modules that can be used in conjunction with Modes 1, 2, or 3: an Autothrottle, a Wavetrain module, and a Model Tracking \& Failures module. The final component in the FCS is a Load Protection System (LPS), which is aimed at preventing the FCS from exceeding the structural limits of the test aircraft. The LPS, Transfer Logic, and Input Selection blocks are always active. Transfers between FCL modes and activation of other functions are controlled by the Transfer Logic block. The Input Selection block smoothes transitions between FCS modes by using linear faders. When a particular mode is engaged, its commands are faded in and the current commands are faded out at the same rate. More detailed description of software considerations for subscale flight testing is provided in [1].

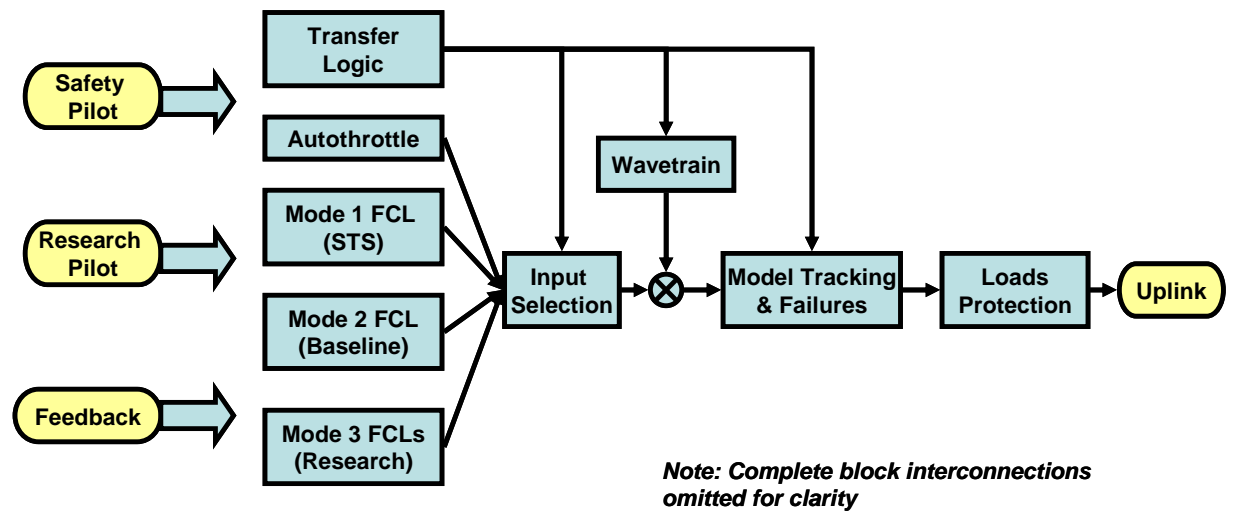

Figure II.3. AirSTAR FCS Flight Control Laws Architecture

The research flight control law, designated as Mode 3 in Figure II.3, was tested in flight in September 2010. This control law was subdivided into two separate research control law blocks, for the purposes of flight test evaluation: the baseline control law plus a CMRAC augmentation and just the baseline control law, which corresponds to LQR+PI architecture. Detailed simulation results and specific control law parameters used for implementation of the baseline plus a CMRAC augmentation are available in [11].

\section{Flight Test Results}

The following material is the results of a flight test of robust baseline LQR+PI controller and of robust baseline LQR+PI controller with CMRAC augmentation flown on AirSTAR aircraft tail number T2 and conducted during the month of September 2010. The flight test objectives were split into two sets of maneuvers: airborne stability and control (S\&C) evaluations, and simulated offset landings which served as a high workload task for handling qualities evaluations. IN each case, the baseline and the research control laws were flown on sequential flight on the same day. While the AirSTAR architecture allows for unlimited switching between different control laws during the same flight, the developed procedures for the control law evaluations called for a single control law to be evaluated during a to The details of tasks flown can be found in the flight test plan [12] and flight cards [13]. Pilot evaluation of command tracking performance in the presence of various emulated faults provided indication of control law 
effectiveness. Control tasks consisted of wavetrain doublet commands superimposed on any pilot stick commands, with the various faults emulated via software. The faults exercised during this flight deployment were: latency injection, aircraft stability degradation, and reduction in control effectiveness, with only the stability degradation cases employed during simulated offset landing evaluations. All flight cards had a target altitude of 900 feet and a target indicated airspeed of 70 to 85 knots (KIAS). The flight cards are segregated such that the two control laws are evaluated with similar inputs for a given card set. For notation sake, throughout this document investigation of cards $\mathrm{XX} / \mathrm{YY}$ indicates that card $\mathrm{XX}$ evaluates the maneuver for the baseline control law, while card YY evaluates similar maneuver for the research control law.

The research control law is a command augmentation system (CAS) with angle of attack (AOA) command in the longitudinal axis and roll rate/angle of sideslip (AOS) command in the lateral/directional axes. During the flight evaluations, the pilot was flying without using the rudder pedals, hence the controller was attempting to hold zero AOS.

\section{A. Nominal (No Failure) Airborne S\&C Results}

The card series (24/38 and 25/39) offered an opportunity to investigate the performance of the control laws (CLAWs) in the absence of latency or failure. These cards injected either angle-of-attack wavetrain (cards 24/38) or roll rate wavetrain (cards 25/39). The longitudinal response of the vehicle using the baseline control law (card 24) and using the research control law (card 38) are shown in Figure III.1 and Figure III.2, respectively. Note that reference model response is included in the baseline plots for comparison of what desired response should look like. Since the baseline operates on an actual aircraft rather than a model, the baseline controller and reference model responses will differ. While it is difficult to directly compare the two responses due to slightly dissimilar input command patterns and because of the turbulence present during card 38, overall both control laws are able to track the reference model while using acceptable surface deflections and activity.
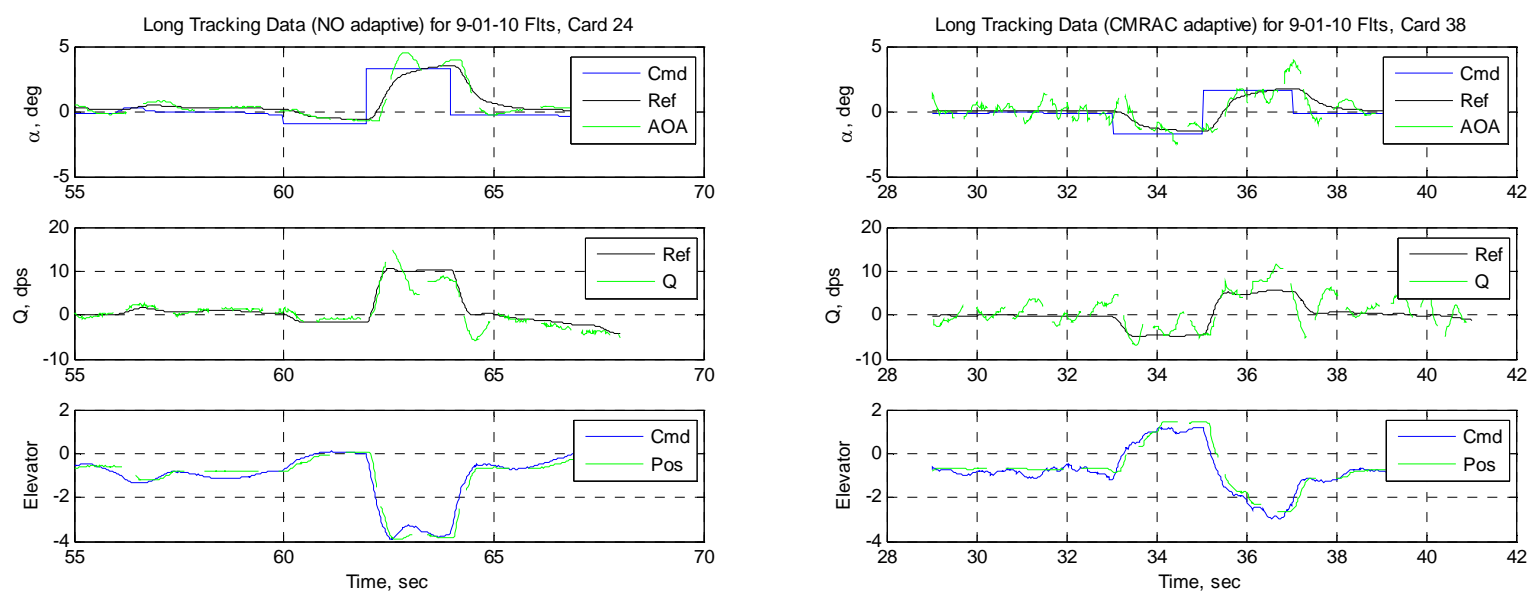

Figure III.1: Longitudinal Tracking, Baseline CLAW, Card 24

Figure III.2: Longitudinal Tracking, Research CLAW, Card 38

In the case of the research control law, the adaptive parameters and predictor performance is shown in Figure III.3, Figure III.4, and Figure III.5. As expected, in the case with no failures, the adaptive parameters show little activity (some mismatch between reference model and actual aircraft is expected in practice, especially in this case when turbulence is encountered), while the predictor shows excellent performance in all axes. The predictor is least effective in the rudder channel, which is expected due to the minimal control activity being employed. However, per design, the predictor in the rudder channel is properly predicting the trend line of the noisy data. Note that the values of the adaptive parameters are problem dependent and the judgment of what constitutes large or small is a subjective opinion of the designer. However, some sense of the appropriate values can be developed based on what a particular parameter tries to estimate. For example, the tracking error in Figure III.3 is related to how well the 
actual AOA is tracking the reference model AOA and for precision tracking numbers less than 1 deg would be considered small. On the other hand, if pitch rate was the tracked variable, numbers an order of magnitude larger, i.e. less than $10 \mathrm{deg} / \mathrm{s}$ would not be considered unreasonable. A similar notion holds for the other parameters. For example, LambdaHat estimates the percent control effectiveness where 1 is $100 \%$ of the reference model control effectiveness; Thetahat is the estimate on the matched uncertainty between reference model and the actual aircraft response, etc. More detailed discussion on the adaptive parameters as well as the entire CMRAC adaptive law is available in [11].
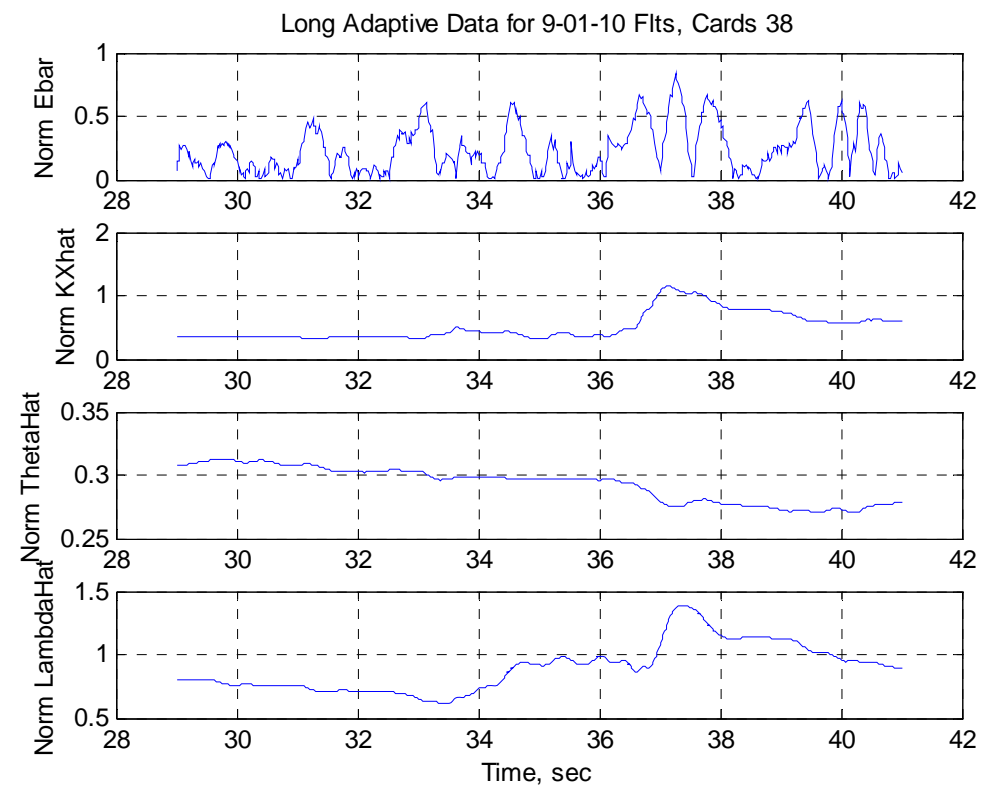

Figure III.3: Longitudinal Adaptive Parameters, Research CLAW, Card 38

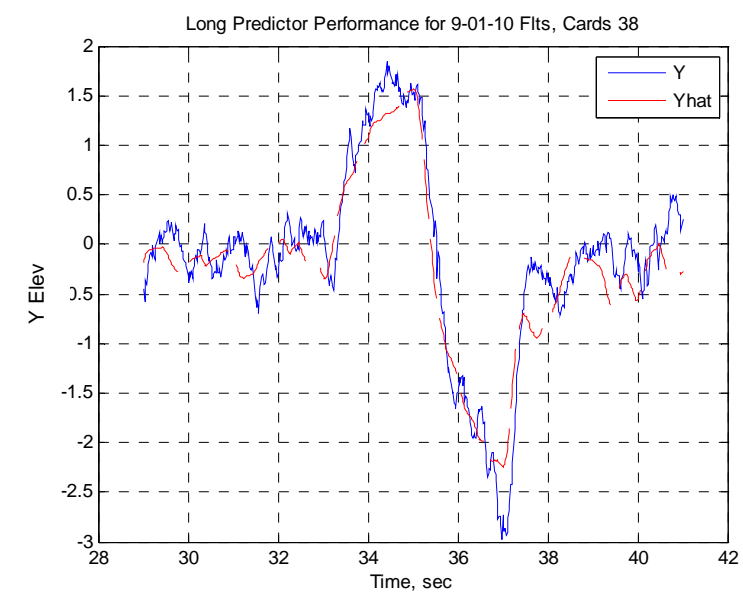

Figure III.4: Longitudinal Predictor ,
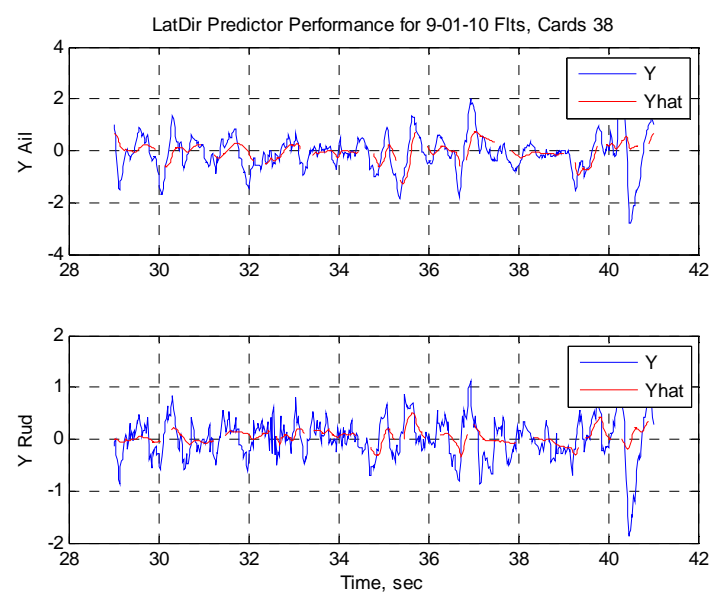

Figure III.5: Lateral/Directional Predictor,

\section{Research CLAW, Card 38}

Performance of the two control laws was also assessed in the frequency domain, by investigating the frequency content of various signals. For example, for cards 24 and 38, the maneuver was AOA tracking, so the corresponding Fast Fourier Transform (FFT) coefficients of the elevator surface signal give insight into the amount of surface activity required by each control law for a similar tracking task. By investigating the FFT coefficients, it is possible to get information regarding control input employed by each control law. The elevator results are shown in Figure III.6, and indicate that for this maneuver the elevator surface activity is similar for both control laws. This is 
expected, since there are no failures and the adaptive augmentation is designed to remain dormant when the system response is nominally similar to the reference dynamics.

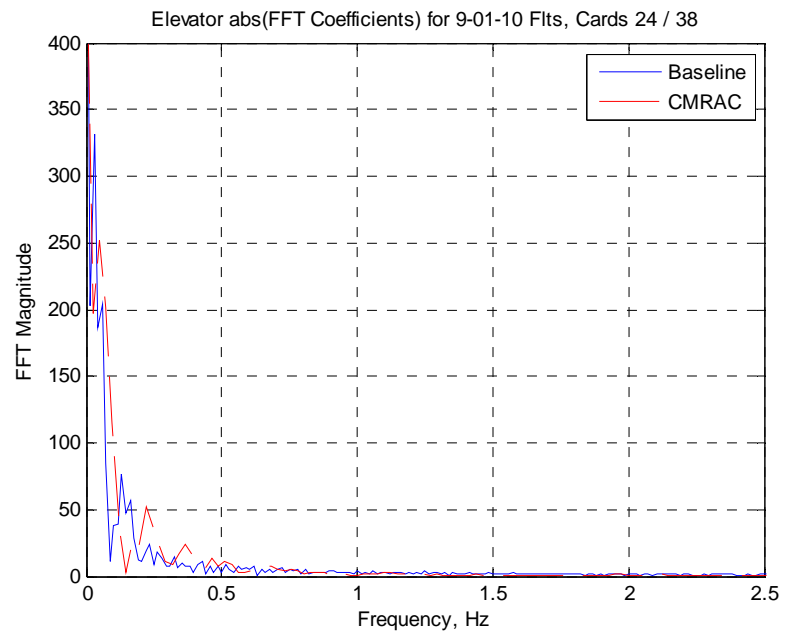

Figure III.6: Coefficients of Elevator Input, Baseline vs. Research CLAW, Cards 24 / 38

In the lateral/directional case, both the aileron and rudder FFT results show increased activity for the research control law, as seen in Figure III.7 and Figure III.8.

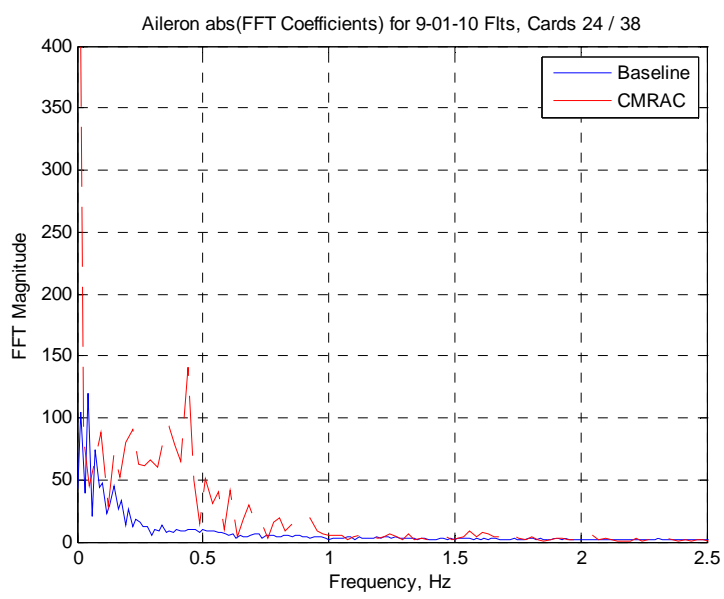

Figure III.7: Coefficients of Aileron Input,

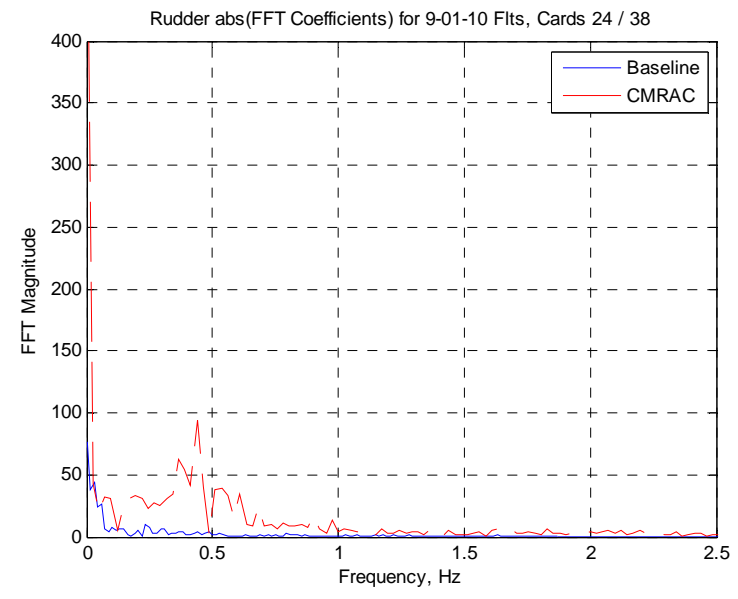

Figure III.8: Coefficients of Rudder Input,

Baseline vs. Research CLAW, Cards 24 / 38

The increased surface activity seen via FFT coefficients is also present in the time histories, as shown in Figure III.9. 

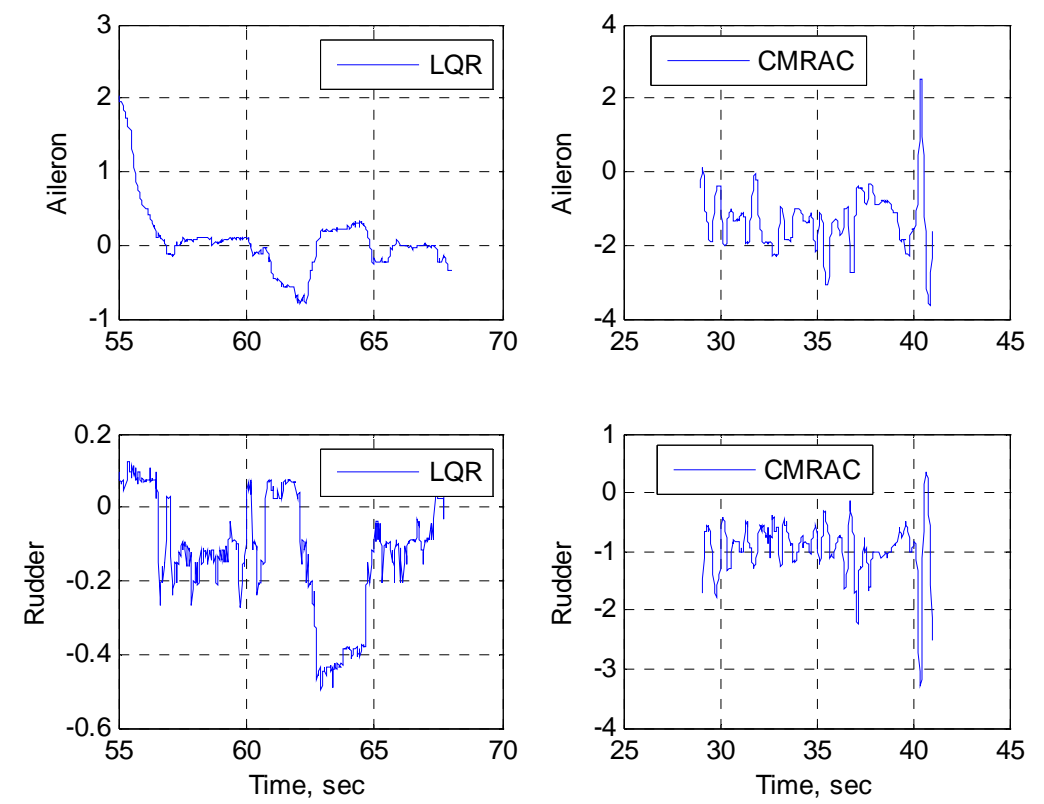

Figure III.9: Lateral/Directional Surface Activity, Baseline (LQR) vs. Research CLAW, Cards 24/ 38

It is difficult to extract conclusions concerning the control activity required, Figure III.7 and Figure III.8, due to the presence of turbulence during card 38. Both the aileron and rudder FFT results have peaks near the same frequency, suggesting that the increase control surface activity is the result of the turbulence rather than additional demands from the research control law. In an effort to further evaluate the research control law performance, card 38 was re-flown during a later flight on 11 September, 2010 during which there was less turbulence (air quality described as "glassy smooth"). The longitudinal tracking results are shown in Figure III.10, and display less oscillatory response than during the first flight test. It does appear that turbulence played a role in the results from the first flight, but even in the presence of turbulence the research control law was able to track AOA command.
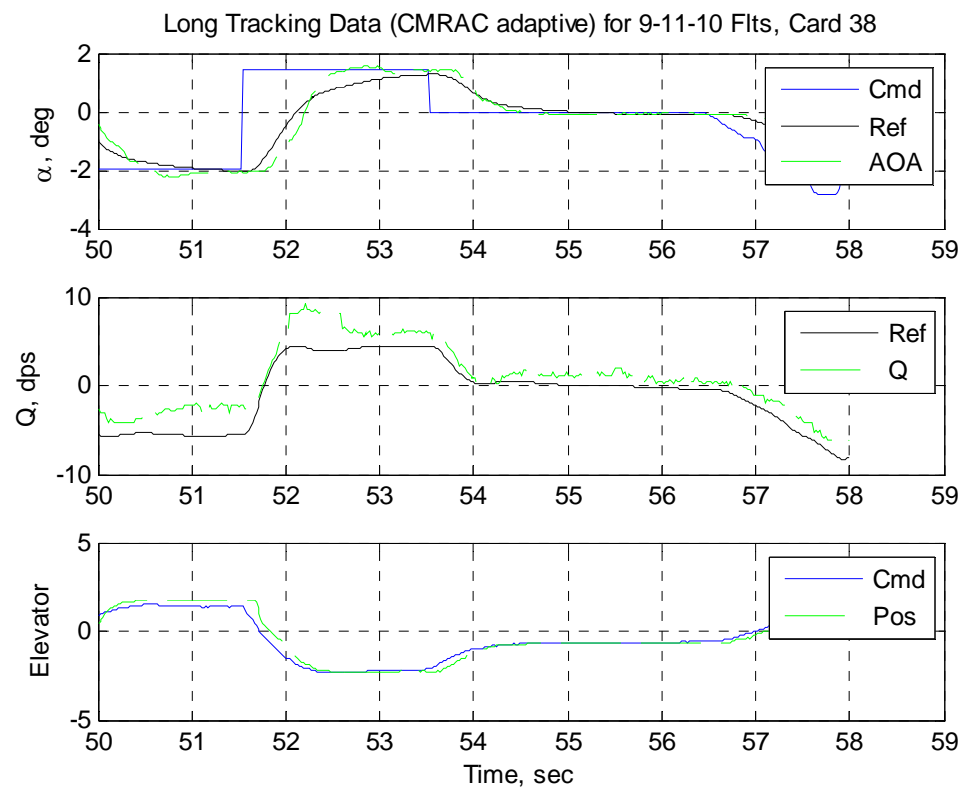

Figure III.10: Longitudinal Tracking, Research CLAW, Card 38, Second Flight

American Institute of Aeronautics and Astronautics 
The FFT coefficients for the elevator, aileron, and rudder surfaces comparing the surface activity for the 1 September flight of card 24 using baseline control law and the 11 September flight of card 38 using research control law are shown in Figure III.11, Figure III.12, and Figure III.13 respectively.

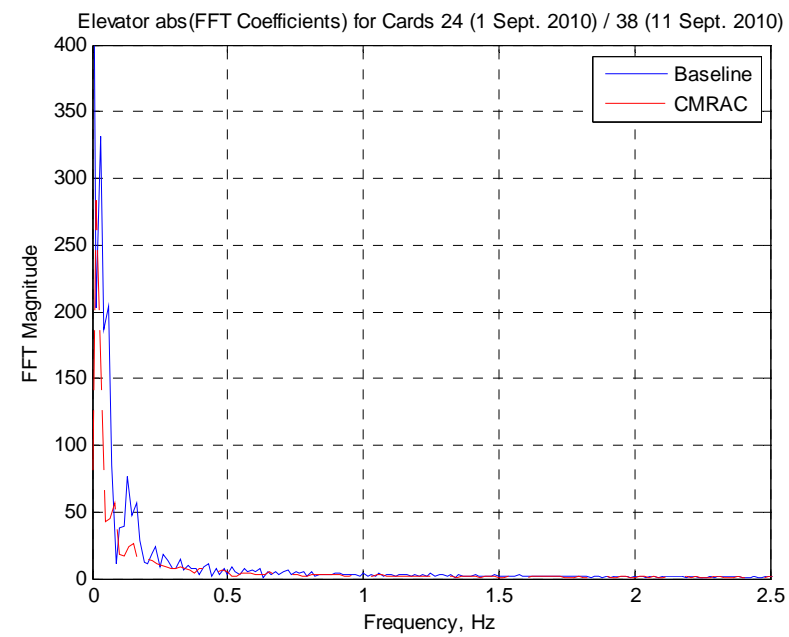

Figure III.11: Coefficients of Elevator Input, Baseline vs. Research CLAW, Cards 24 / 38

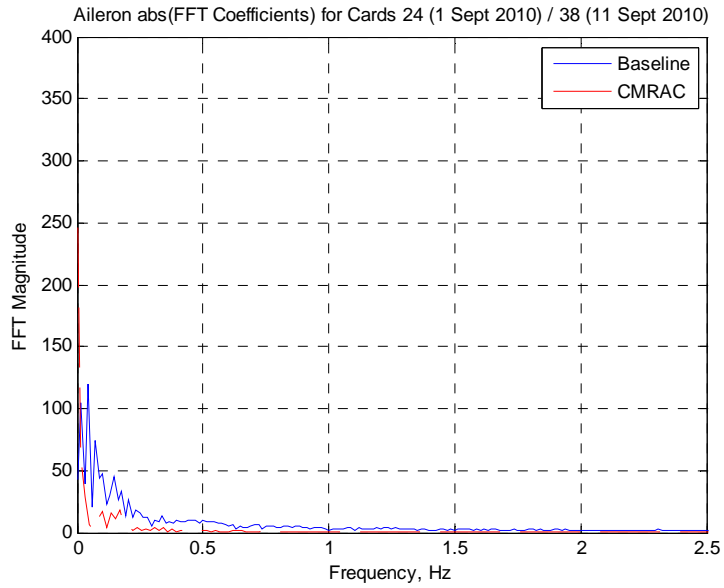

Figure III.12: Coefficients of Aileron Input,

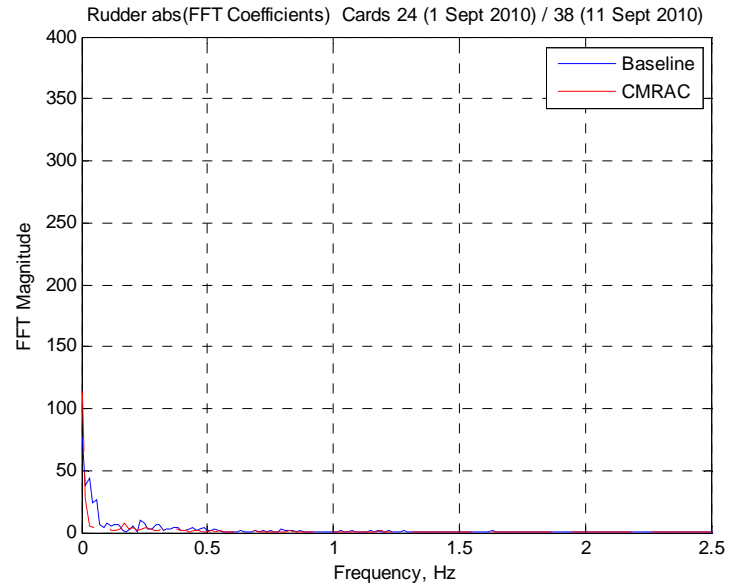

Figure III.13: Coefficients of Rudder Input,

Baseline vs. Research CLAW, Cards 24 / 38

The results show that in the absence of turbulence, the research control law is employing less control surface activity in all axes while achieving the tracking task, illustrating the beneficial effects of the CMRAC architecture.

The data for cards 25/39 are similar to the previous results, albeit for the lateral/directional axes following roll rate command input. Because of the similarity in response and lack of pilot comments, detailed results are not included here. The roll rate tracking response is included in Figure III.14 and Figure III.15 for comparison with subsequent maneuvers in which failures are present. The system response becomes oscillatory throughout the maneuver.

The FFT coefficient results for cards 25/39 are not included for brevity and because they show minimal difference between the baseline and research control laws. This further suggests that the increased control activity seen for the aileron and rudder surfaces in cards $24 / 38$ are likely due to turbulence effects. This is expected, since in both of these card sets there are no failures present and the adaptive augmentation is designed to have no input if baseline aircraft response matches the reference model. 

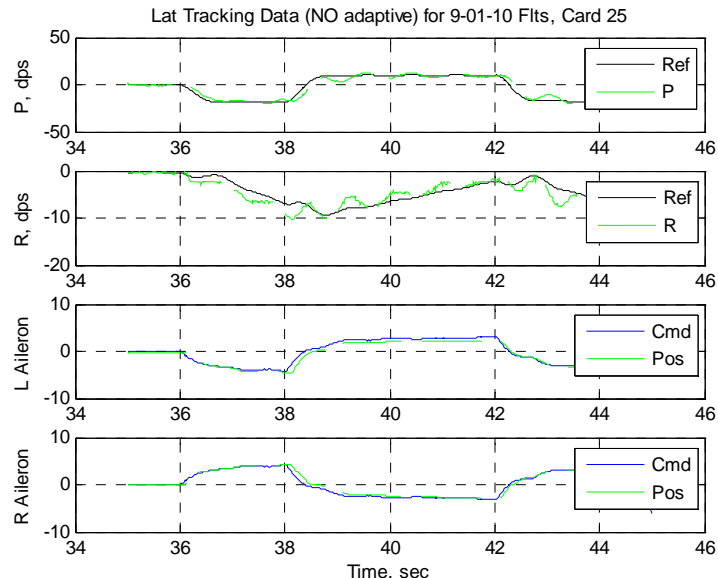

Figure III.14: Lateral/Directional Tracking, Baseline CLAW, Card 25
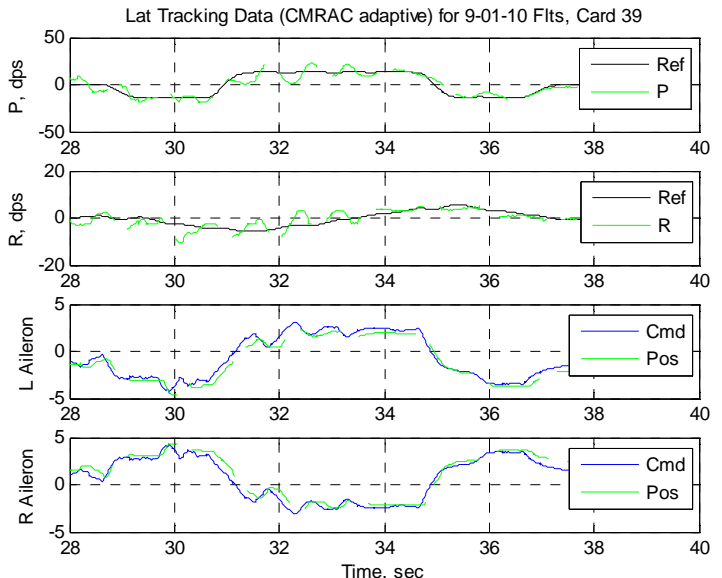

Figure III.15: Lateral/Directional Tracking, Research CLAW, Card 39

\section{B. Airborne S\&C Results with Latency Injection}

The card set 22/36 employed an AOA wavetrain in the presence of latency fault in the system control channels. The latency delay began at a value of 0.02 seconds and was incremented in values of 0.005 seconds of delay every 5 seconds of flight time until the system exhibits instability. The initial and incremental values were chosen to provide gradual loss of robustness in order to explore the control law performance degradation behavior. The latency fault was carried through the turns, unlike the stability degradation faults. An AOA and a roll rate doublet wavetrains were injected on alternate straight legs as range and flight condition permitted. In summary, the baseline control law could tolerate a 0.070 second latency fault and the research control law could tolerate 0.065 second latency fault before onset of instability.
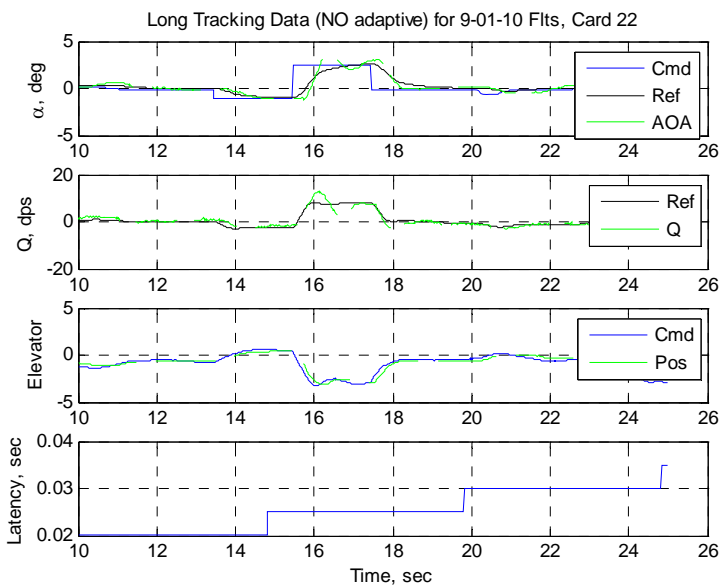

Figure III.16: Longitudinal Tracking, Baseline CLAW, Card 22
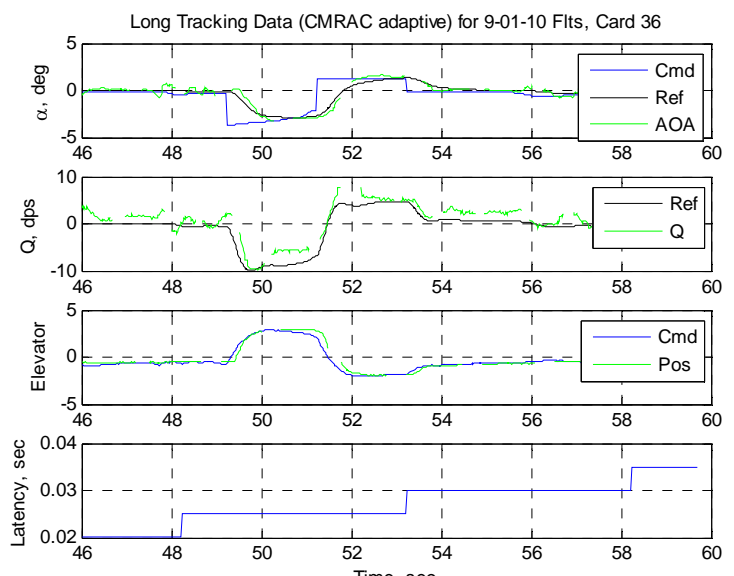

Figure III.17: Longitudinal Tracking, Research CLAW, Card 36

The longitudinal response of the baseline flight control law is shown in Figure III.16. The response shows that the reference model (ideal closed-loop response) tracks the external angle-of-attack command, while the actual aircraft response tracks with significant oscillation. The oscillation is due to the latency in the system, which is 0.025 seconds at the time the maneuver was performed. A similar maneuver was performed with the research flight control law. During the flight of the research control law (Flight 38), there was increased turbulence compared to the baseline control law flight, which is seen in the aircraft response. 
The longitudinal response of the research control law is shown in Figure III.17. The effect of the increased turbulence can be seen in the time history during the lead-in to the angle-of-attack maneuver. It can be noted that during the AOA maneuver, the response of the research control law is much less oscillatory and tracks the reference model more closely than the baseline control law, for a similar latency value of 0.025 seconds. The baseline control law does have the disadvantage of the latency value stepping from 0.02 to 0.025 in close proximity to the doublet reversal. Further inspection of the behavior of the research control law adaptive parameters shows that CMRAC is able to improve tracking without large adaptive parameter variations, as seen in Figure III.18. Once again, note that the reference to the magnitude of adaptive parameters is problem specific and is subjective per control designer; however, if one sees numbers that are orders of magnitude larger than those observed for a nominal aircraft, then a claim can be made that a large adaptive parameter variation has occurred.
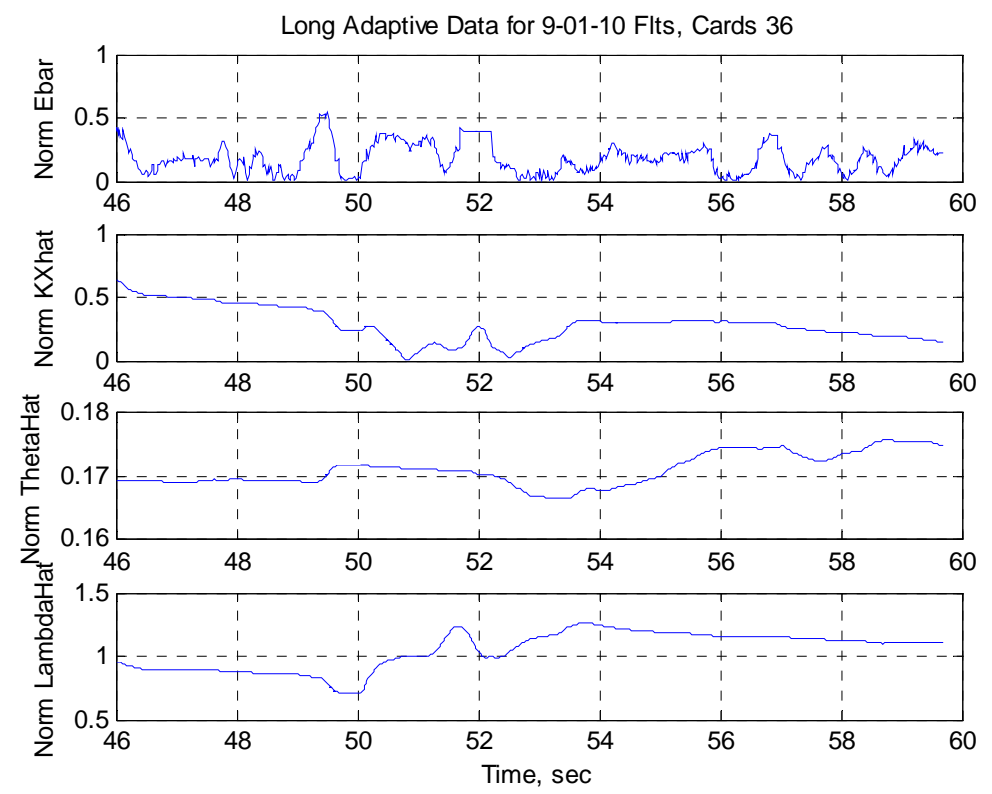

Figure III.18: Longitudinal Adaptive Parameters, Research CLAW, Card 36

Although the performance of the research control law in the presence of latency shows improvement over the baseline control law, care must still be taken in the design and implementation of adaptive augmentation. While there is little activity in the lateral/directional axes during the maneuver, as seen in Figure III.19 the adaptive parameters in these axes remain active. In particular, adaptive parameters labeled "THETAHAT" show behavior typical of parameter drift, which is a known concern in adaptive systems and which can lead to instability if not properly accounted for. In this design, known design robustification techniques such as deadzone and projection operator were included, but the response indicates that additional analysis and tuning is recommended.

Once again investigating the FFT coefficients of the control surface signals gives insight into the amount of surface activity required by each control law for a similar tracking task. These results, shown in Figure III.20 and Figure III.21, confirm that the research control law augmentation is able to improve tracking performance in the presence of uncertainty, in this case latency, while requiring reduced control input.

It is interesting to note that the benefit of the research control law is not restricted to the axis subjected to commands in this case. The FFT coefficients corresponding to the aileron surface input, shown in Figure III.21, also show significant reduction in control activity, especially at lower frequencies. This indicates the research control law is providing improved tracking while using reduced control activity, and also using reduced control input in axes in which the primary control task is regulation. The rudder surface FFT coefficients show minimal differences and are therefore not included here. 

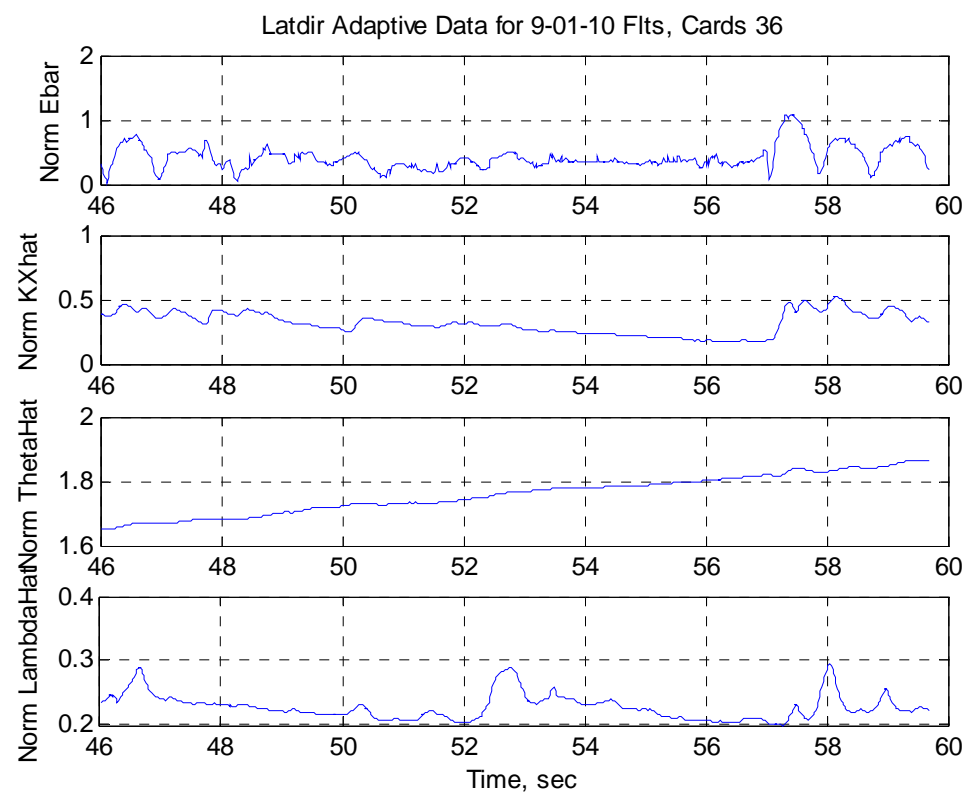

Figure III.19: Lateral/Directional Adaptive Parameters, Research CLAW, Card 36

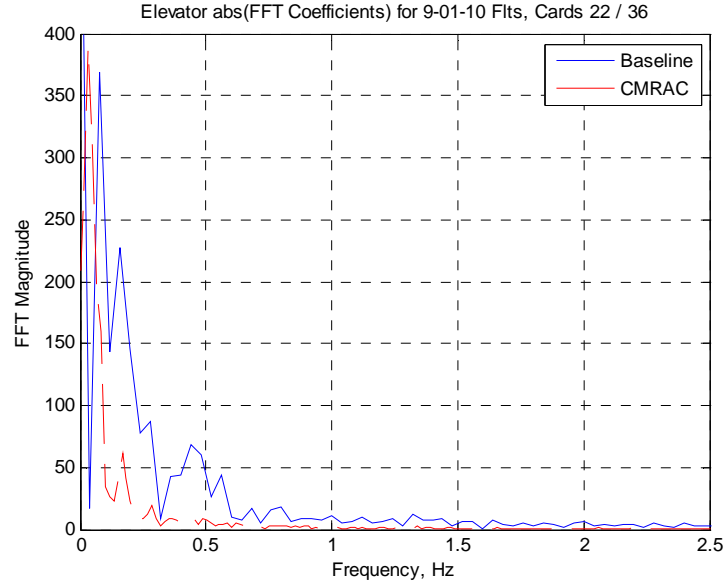

Figure III.20: FFT Coefficients of Elevator Input,

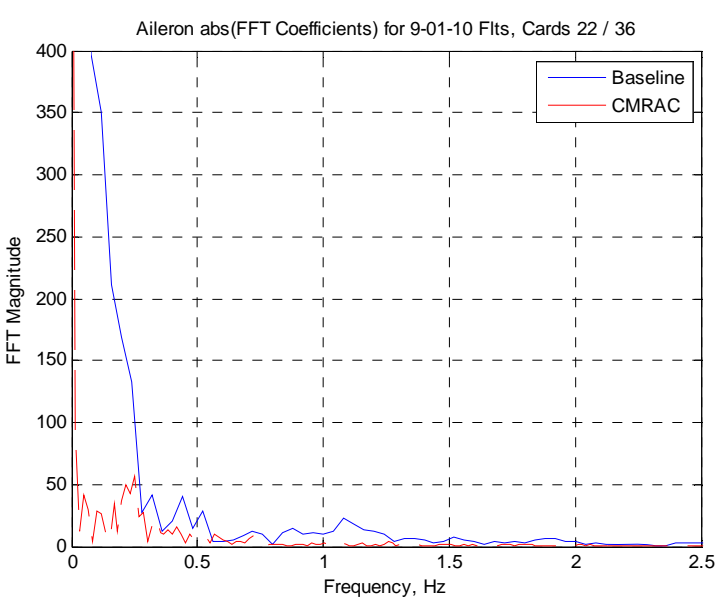

Figure III.21: FFT Coefficients of Aileron Input,

Baseline vs. Research CLAW, Cards 22 / 36

\section{Airborne S\&C Results with Stability Degradation (Longitudinal)}

The next set of cards begins a gradual build-up routine in which the pitch and roll channels are destabilized via reduction in $C_{m \alpha}$ and $C_{l p}$ respectively. Cards 26 (Baseline) and 40 (Research) are pitch wavetrain maneuvers with $50 \%$ reduction in $C_{m \alpha}$ and $C_{l p}$, and the reduction is increased by $25 \%$ in each consecutive card pair, culminating in $100 \%$ reduction in $C_{m \alpha}$ and $C_{l p}$ in cards 30/44. The destabilization in $C_{m \alpha}$ is achieved by removing the inboard elevator segments from the feedback control path and scheduling them with AOA, while the reduction in $C_{l p}$ is achieved by inserting spoiler surface motion scheduled with roll rate (spoiler surfaces are not commanded by control laws). In the process, the longitudinal control authority is also reduced by $50 \%$. As the spoilers are not used for roll control, no loss of lateral control authority results. 
For cards 26/40, the longitudinal aircraft response to AOA wavetrain with $50 \%$ reduction in $C_{m \alpha}$ and $C_{l p}$ is shown in Figure III.22 and Figure III.23 respectively. In both cases, the inboard elevator deflection response shows the simulated reduction in $C_{m \alpha}$, while the outboard elevator follows the control law command. The spoiler surface motion injected to achieve the reduction in $C_{l p}$ is not included for brevity. The general response of the research control law contains less overshoot than the baseline control law, however due to the increased turbulence it is difficult to make a definitive statement regarding the efficacy of the augmentation.
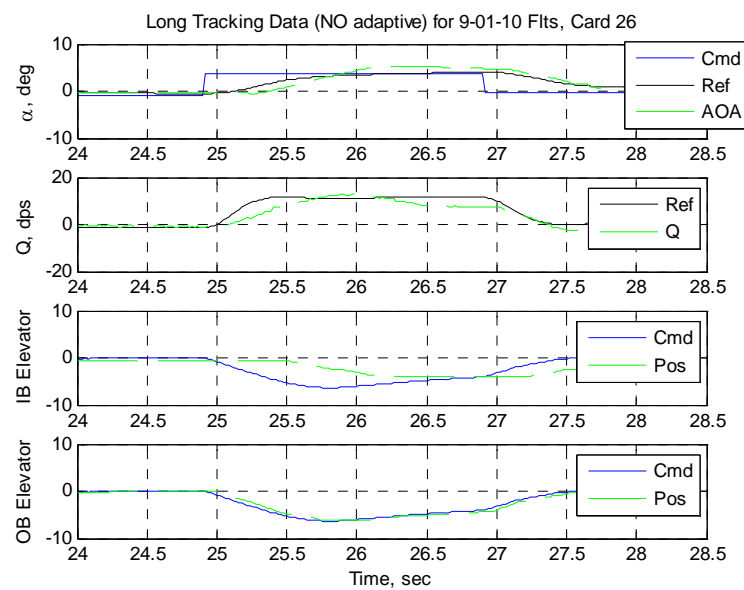

Figure III.22: Longitudinal Tracking, Baseline CLAW, Card 26
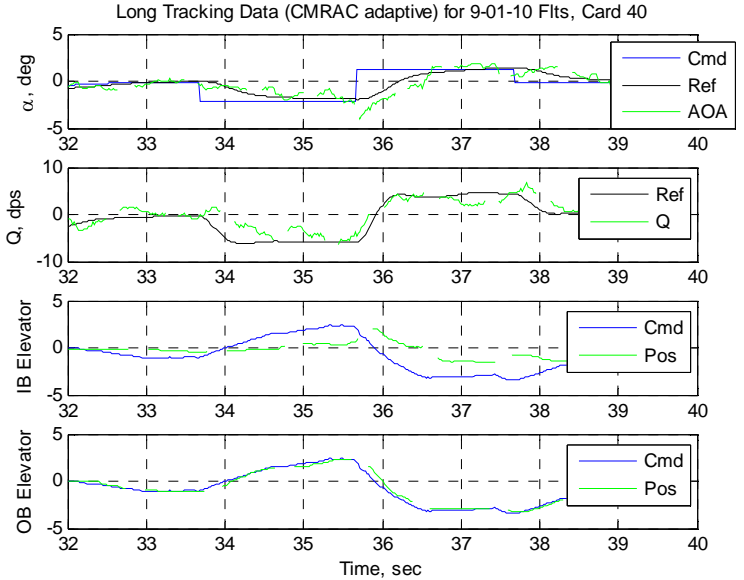

Figure III.23: Longitudinal Tracking, Research CLAW, Card 40

The longitudinal adaptive and predictor performances are shown in Figure III.24 and Figure III.25, respectively. These plots show that despite the turbulence present and the change in the aircraft characteristics, the adaptive augmentation is responding per design to maintain tracking of the reference dynamics and the predictor matches the trend-line of the system response. There were no pilot comments for either card.
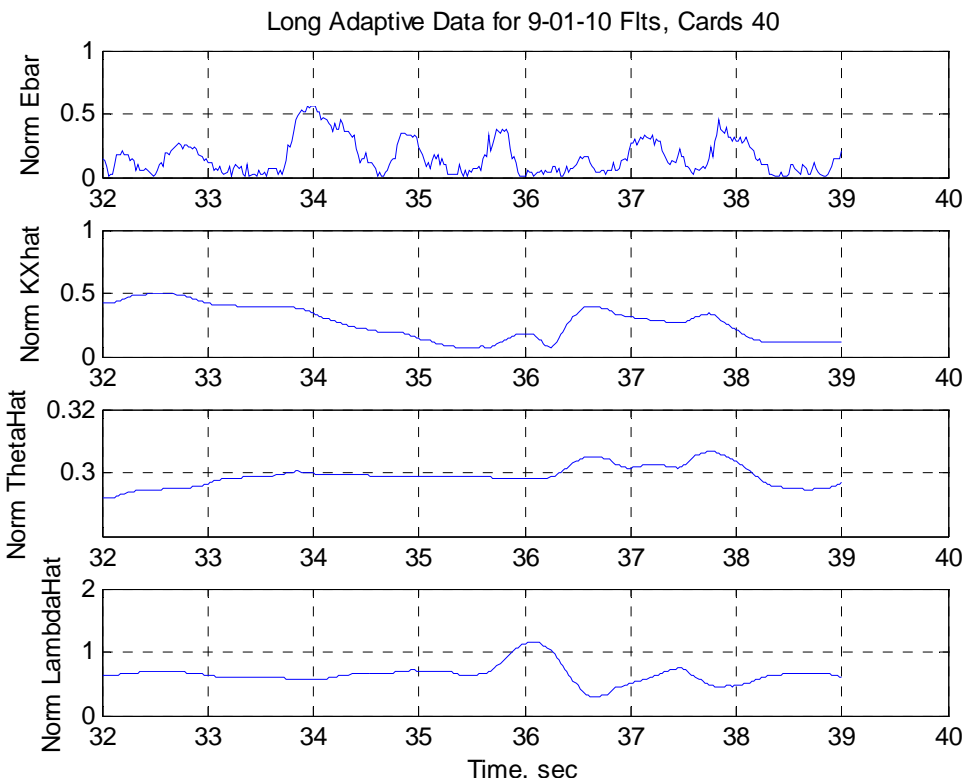

Figure III.24: Longitudinal Adaptive Parameters, Research CLAW, Card 40

12

American Institute of Aeronautics and Astronautics 


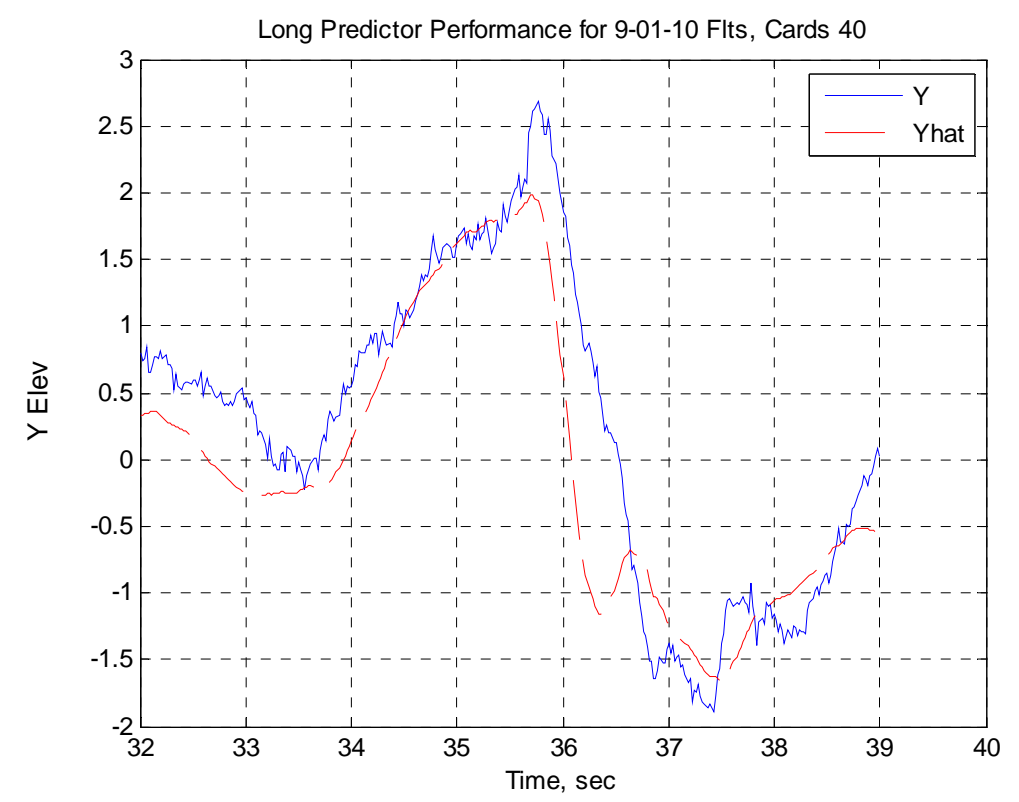

Figure III.25: Longitudinal Predictor Performance, Research CLAW, Card 40

For cards 28/42, the aircraft longitudinal response to AOA wavetrain with $75 \%$ reduction in $C_{m \alpha}$ and $C_{l p}$ is shown in Figure III.26 and Figure III.27, respectively. Once again, the reduction in $C_{m \alpha}$ is seen in the inboard elevator surface response. The longitudinal response again shows less pronounced overshoot in the research control law case, with similar elevator deflection and activity in both cases. The pitch rate response appears slightly more responsive in the research control law maneuver, although the turbulence makes true comparison difficult.
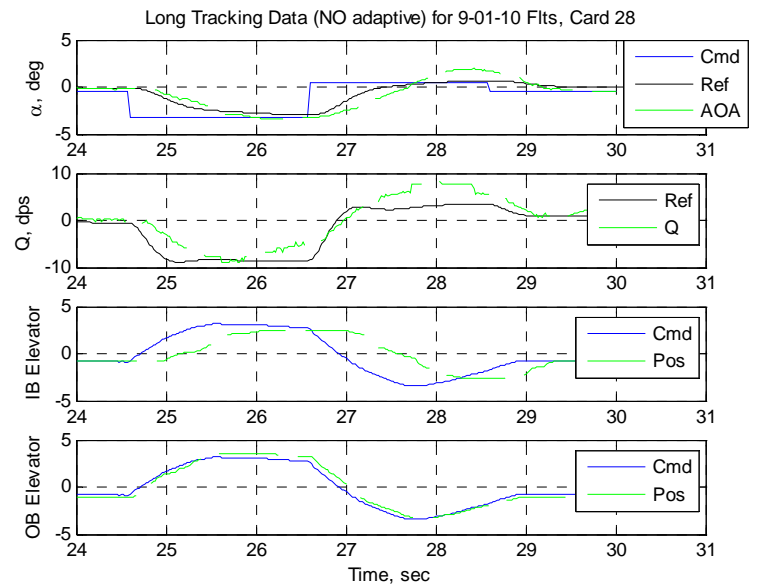

Figure III.26: Longitudinal Tracking, Baseline CLAW, Card 28
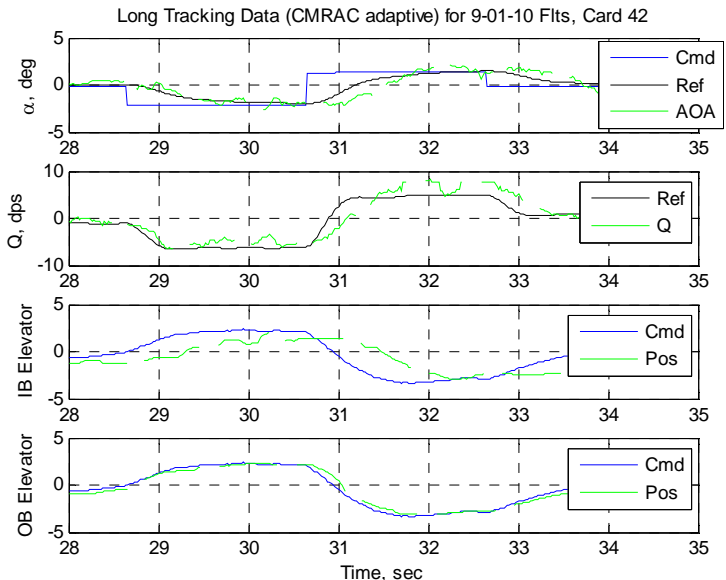

Figure III.27: Longitudinal Tracking,

Research CLAW, Card 42

At this level of $C_{m \alpha}$ and $C_{l p}$ destabilization, both the baseline and research control law received pilot comments of roll axis limit cycle oscillations during the pitch doublet maneuver. The corresponding roll axis tracking information for the cards for each axis are shown in Figure III.28 and Figure III.29 respectively. Inspection of the roll response indicates that the research control law is generating more roll rate excursion and control surface activity in the roll axis during the pitch maneuver. Note that the yaw rate measurement in the baseline control law 
case may be corrupted, however the general magnitude difference between roll and yaw rates in the two control laws is sufficient to show a significant change in behavior.
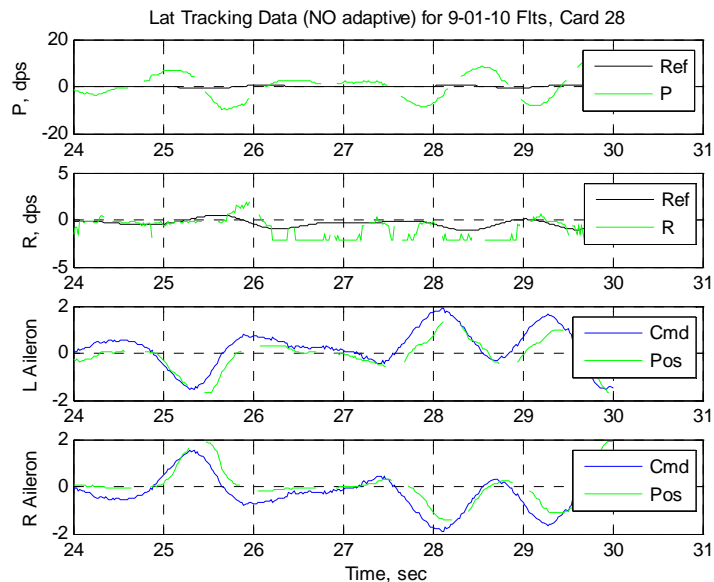

Figure III.28: Lateral/Directional Tracking,

Baseline CLAW, Card 28
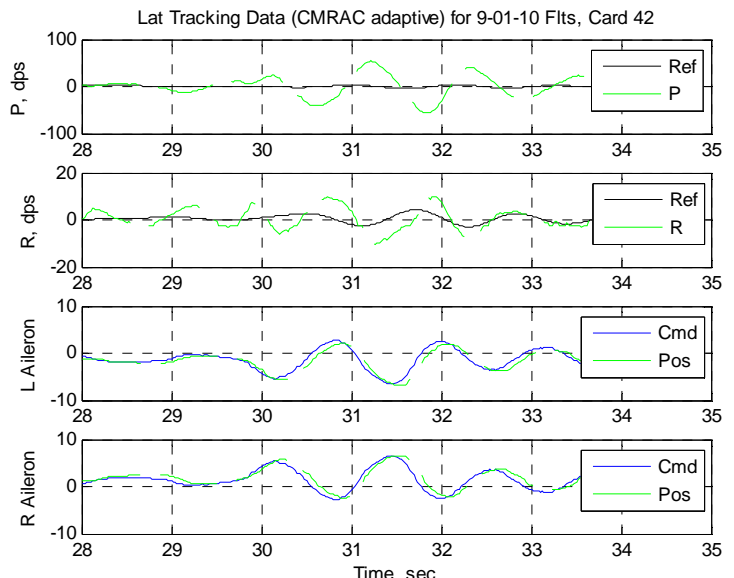

Figure III.29: Lateral/Directional Tracking,

Research CLAW, Card 42

The adaptive parameters for both axes for card 42 are shown in Figure III.30 and Figure III.31, respectively. The results show that the longitudinal axis adaptive system is working to maintain tracking with small increments in the adaptive parameters, as expected. The lateral/directional adaptive parameters show more activity and greater magnitude.
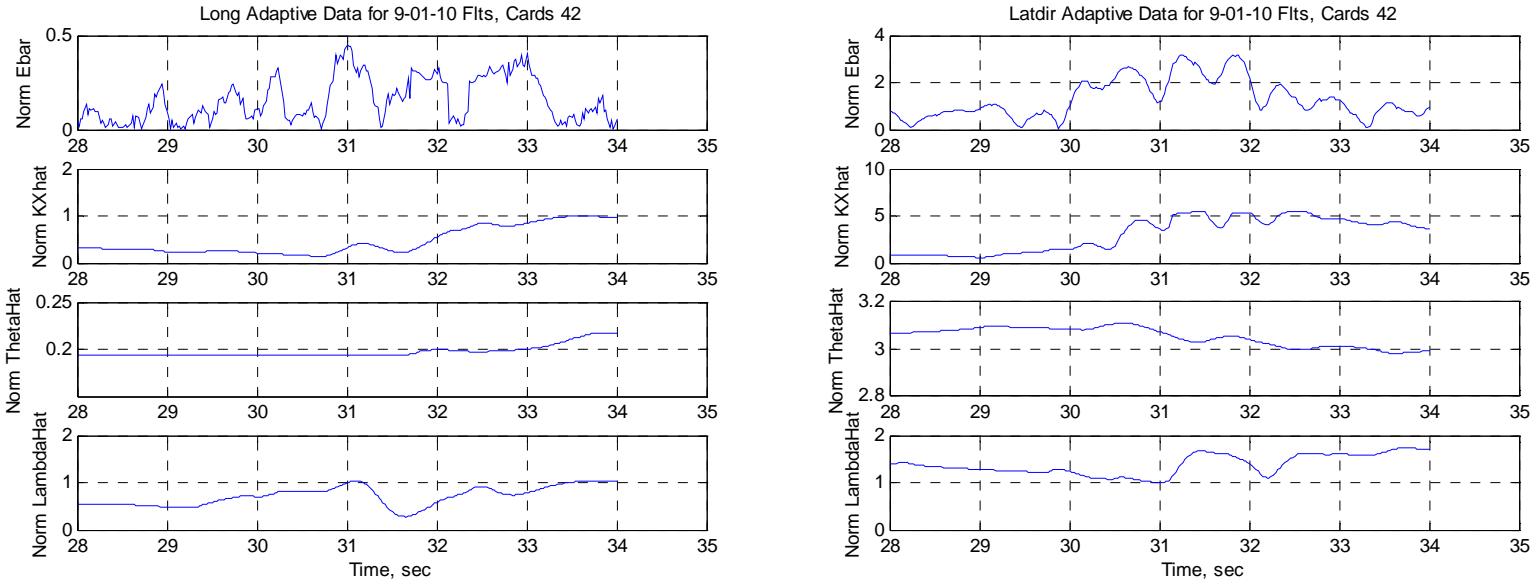

Figure III.30: Longitudinal Adaptive

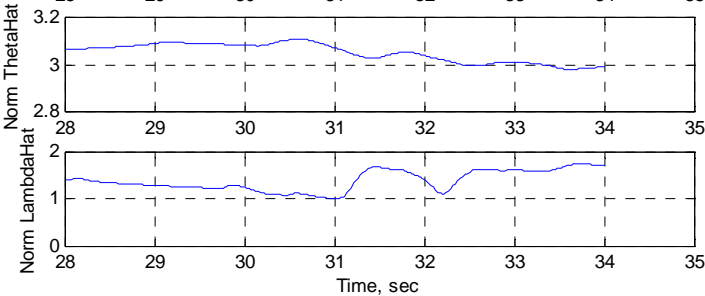

Figure III.31: Lateral/Directional Adaptive

Parameters, Research CLAW, Card 42

The behavior of the lateral/directional predictor is shown in Figure III.32. It is interesting to note that the predictor generally does a good job of predicting the system output behavior, however; in both the aileron and rudder channels the predictor does not perform well in the negative direction. Whether this behavior is a result of the roll limit cycle and resulting rapid variation in vehicle rates, or whether this behavior is a contributing cause to the roll limit cycle warrants further investigation. 

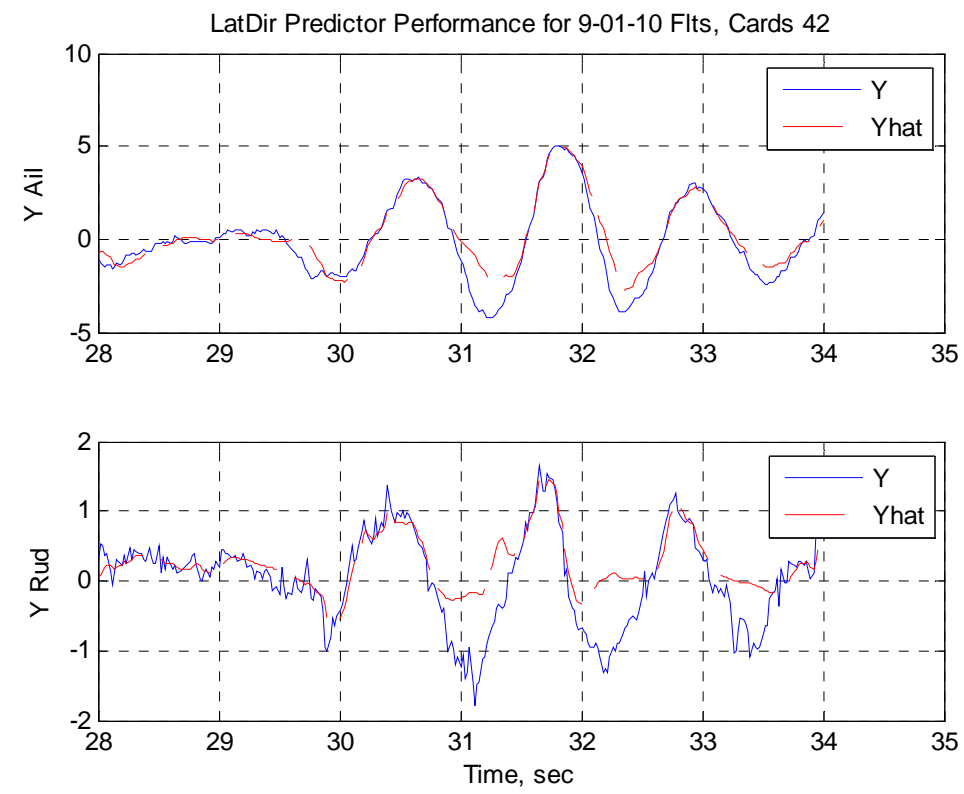

Figure III.32: Lateral/Directional Predictor Performance, Research CLAW, Card 42

Cards 30/44 show data with injected AOA wavetrain and with $100 \%$ reduction in $C_{m \alpha}$ and $C_{l p}$, i.e. neutrally stable aircraft, and resulting in KIO (knock it off) call due to roll oscillations for both the baseline and research control laws. The longitudinal data for the maneuver as completed are shown in Figure III.33 and Figure III.34.
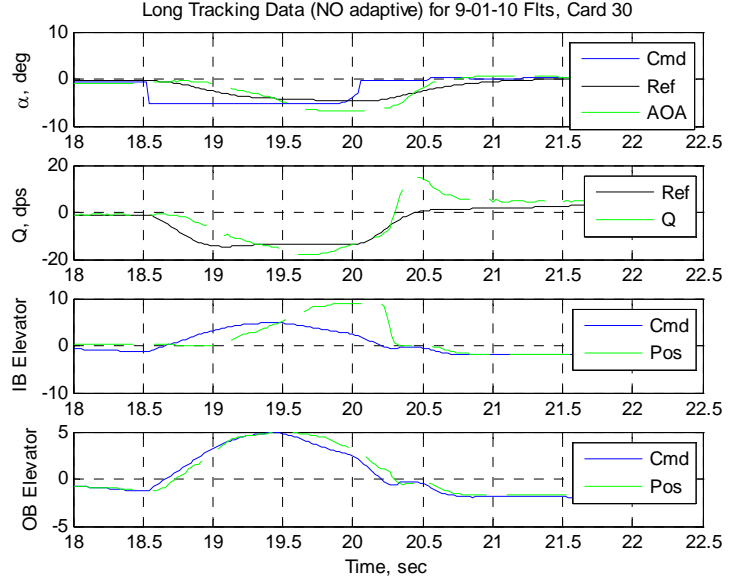

Figure III.33: Longitudinal Tracking,

Baseline CLAW, Card 30
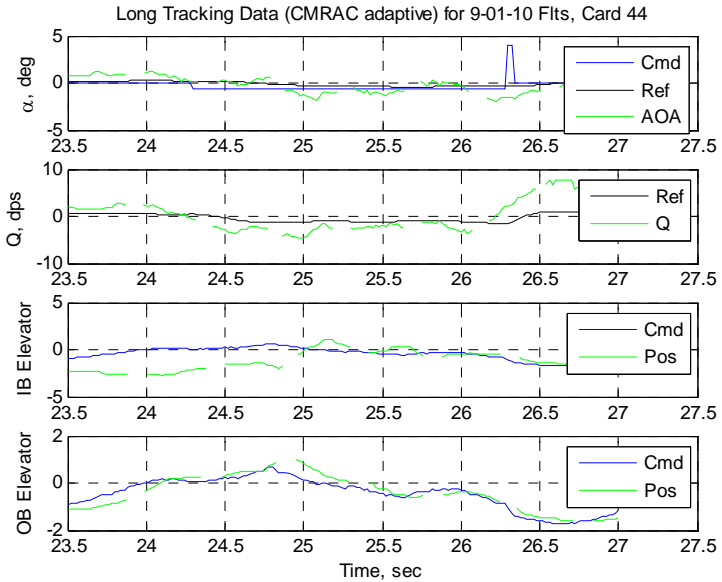

Figure III.34: Longitudinal Tracking, Research CLAW, Card 44

The baseline control law completed part of a doublet, while the research control law did not complete the doublet prior to KIO call. Neither control law shows significant problems in the longitudinal axis, but inspection of the lateral/directional responses for the baseline control law in Figure III.35 and Figure III.36 show significant roll rate oscillations. 


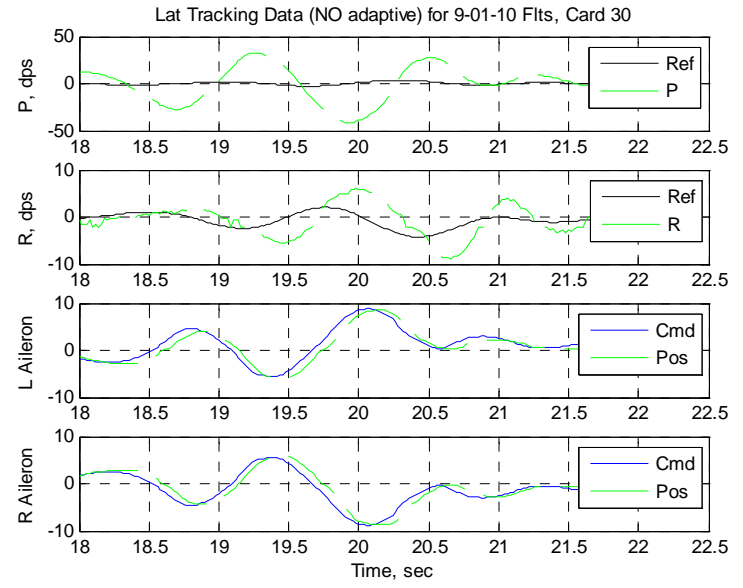

Figure III.35: Lateral Tracking,
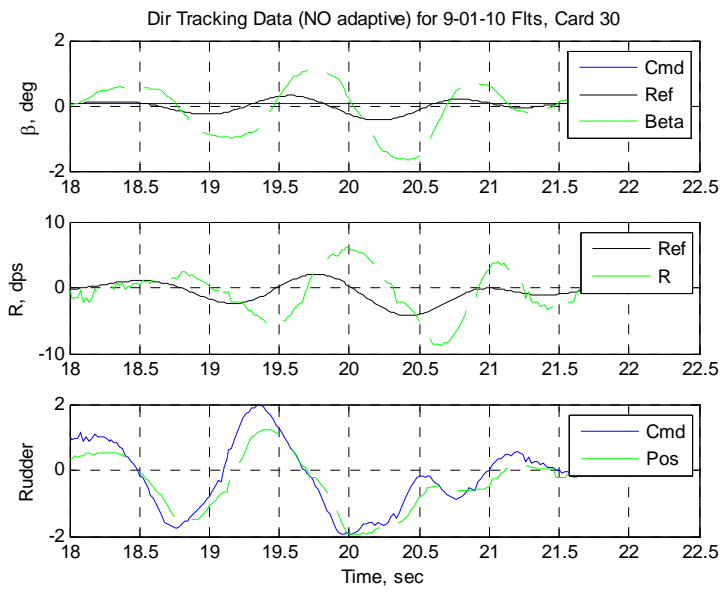

Figure III.36: Directional Tracking,

\section{Baseline CLAW, Card 30}

Both the aileron and rudder surfaces are active during roll rate oscillations. It should be noted that on this vehicle the influence of the rudder on the lateral/directional dynamics will overpower that of the ailerons for significant deflections. This is likely the cause of the more severe roll oscillation in the case of the research control law, as seen in Figure III.37 and Figure III.38.
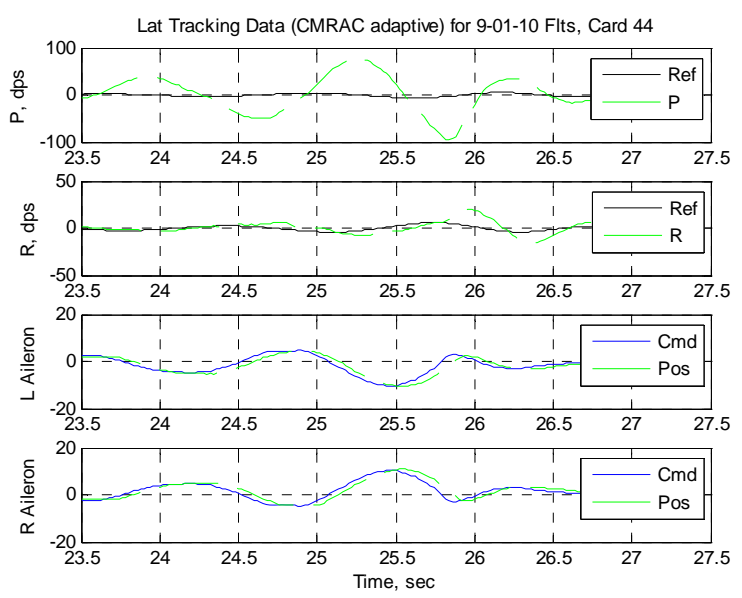

Figure III.37: Lateral Tracking,
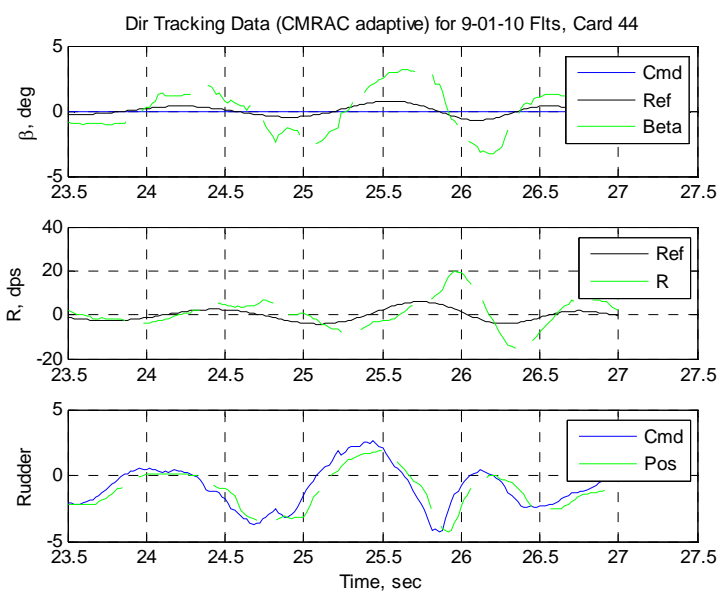

Figure III.38: Directional Tracking,

\section{Research CLAW, Card 44}

The responses show that while aileron deflection is of similar magnitude between the two control laws, the rudder deflection is larger in the case of the research control law and this is a likely cause of the larger roll oscillations. It is interesting to note that neither the lateral/directional adaptive parameters nor the lateral/directional predictor results contain large gain changes or excessive dynamics, as shown in Figure III.39 and Figure III.40. 

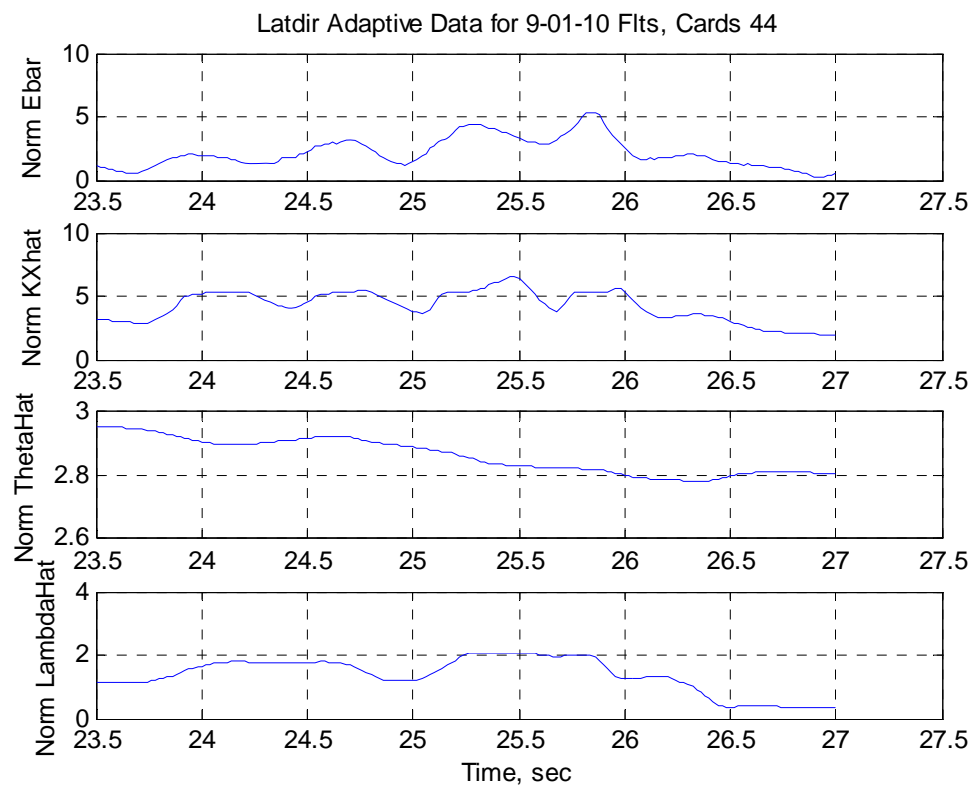

Figure III.39: Lateral/Directional Adaptive Parameters, Research CLAW, Card 44

From the results, it appears that the lateral/directional research control law may need to be tuned to possess more damping or to be less sensitive. However, it bears noting that the baseline control law also resulted in a KIO call, and that the flight including adaptive augmentation took place in significantly more turbulence, as evidenced in the previous cards of these flights as well. Furthermore, offline simulations expected a KIO call in the case of this maneuver.
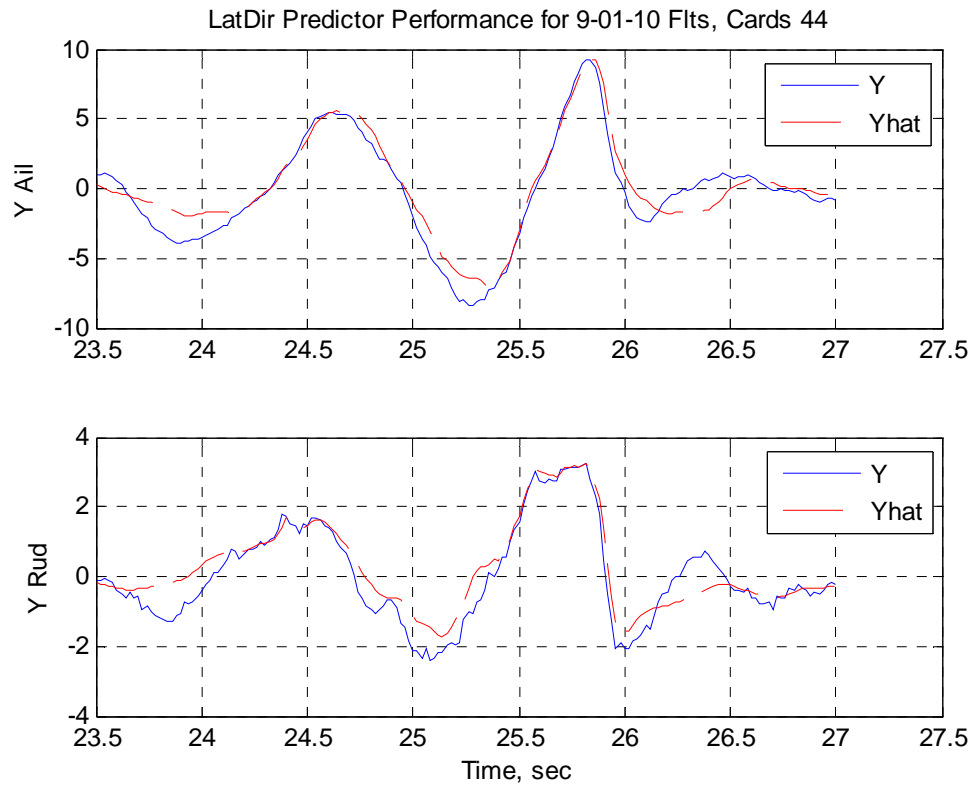

Figure III.40: Lateral/Directional Predictor Performance, Research CLAW, Card 44

\section{Airborne S\&C Results with Stability Degradation (Lateral/Directional)}

Similarly to the destabilization failure cards in the longitudinal axis, a similar set of evaluations are performed in the lateral/directional axes. Cards $27 / 41$ are roll rate wavetrain maneuvers with $50 \%$ reduction in $C_{m \alpha}$ and $C_{l p}$, 
and the reduction is increased by $25 \%$ in each consecutive card pair, culminating in $100 \%$ reduction in $C_{m \alpha}$ and $C_{l p}$ for cards $31 / 45$.
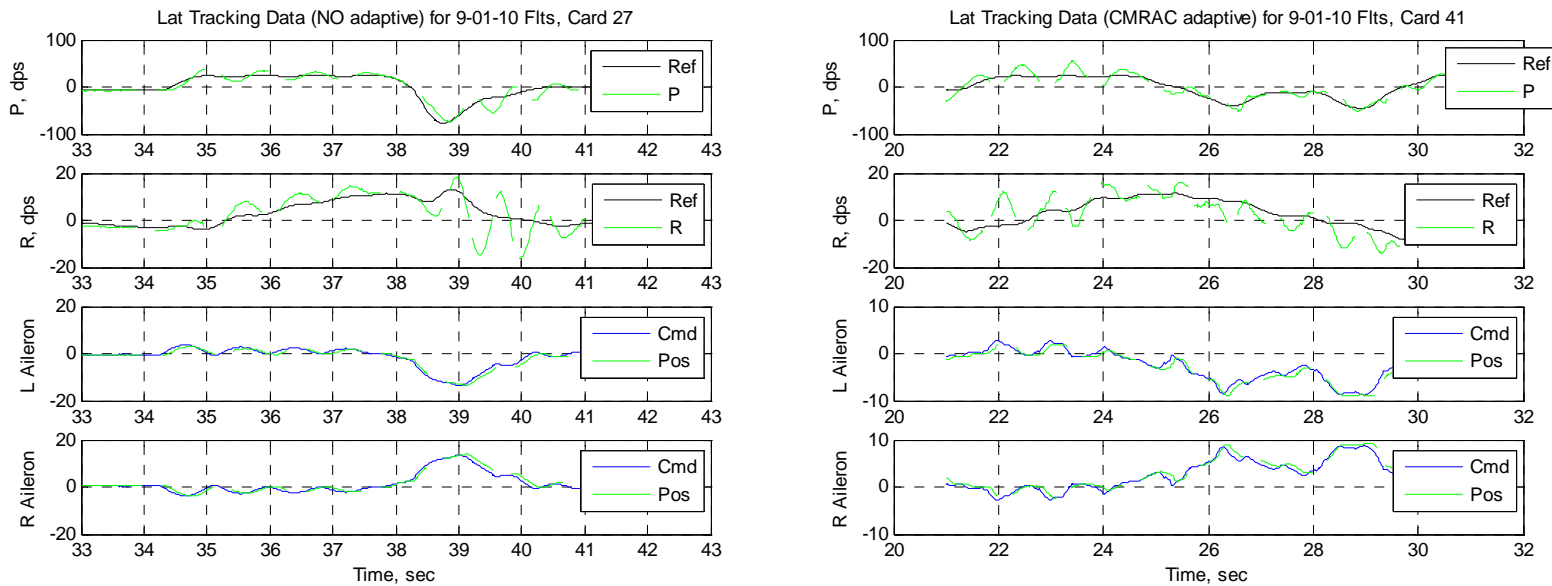

Figure III.41: Lateral Tracking, Baseline CLAW, Card 27

Figure III.42: Lateral Tracking, Research CLAW, Card 41

The lateral aircraft response to roll rate wavetrain with $50 \%$ reduction in $C_{m \alpha}$ and $C_{l_{p}}$ is shown in Figure III.41 (card 27) and Figure III.42 (card 41). In this case, there were no pilot comments for card 27, while for card 41 the pilot reported limit cycle oscillation in roll. However, it is difficult to see a limit cycle oscillation in the response of card 41. Turbulence is certainly more severe in this case, as in previous comparisons between flights 37 and 38 , as seen in the unsteady approach to the roll rate maneuver. Once the roll rate doublet maneuver is initiated, both the baseline and research control laws are able to track the reference model with slightly underdamped response. The baseline control law appears to have a less favorable yaw response based on the yaw rate trace, and uses more control surface deflection than the research control law. These characteristics are also seen in the directional response of the aircraft, shown in Figure III.43 and Figure III.44. The directional response traces confirm that the research control law appears more oscillatory in general, although how much of this is due to increased turbulence is not readily clear. The baseline control law shows more rudder activity and noticeable oscillation on the reversal of the roll rate wavetrain, but this response did not appear objectionable to the pilot based on comments.
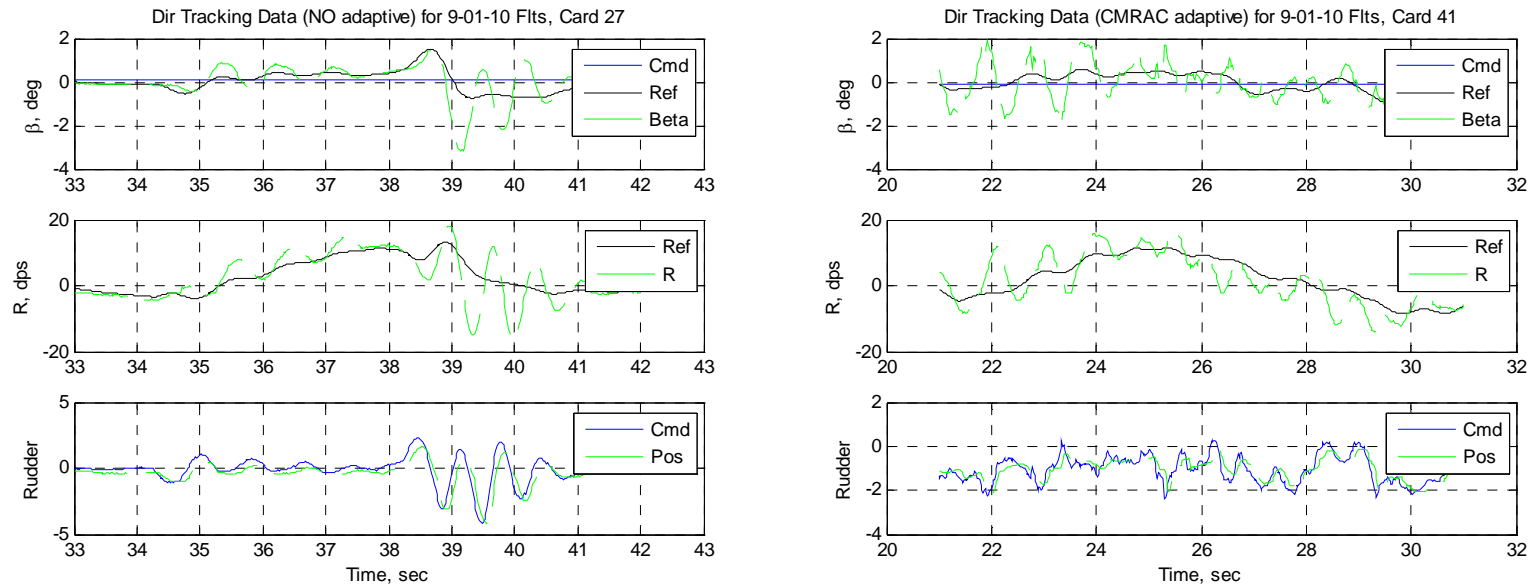

Figure III.43: Directional Tracking, Baseline CLAW, Card 27 Research CLAW, Card 41

18

American Institute of Aeronautics and Astronautics 
Inspection of the lateral/directional adaptive parameters shown in Figure III.45 indicate that the research CLAW augmentation is acting to maintain reference model tracking, but without large adaptive gain changes or severe dynamic changes. This is an indication that the research control law is performing per design.
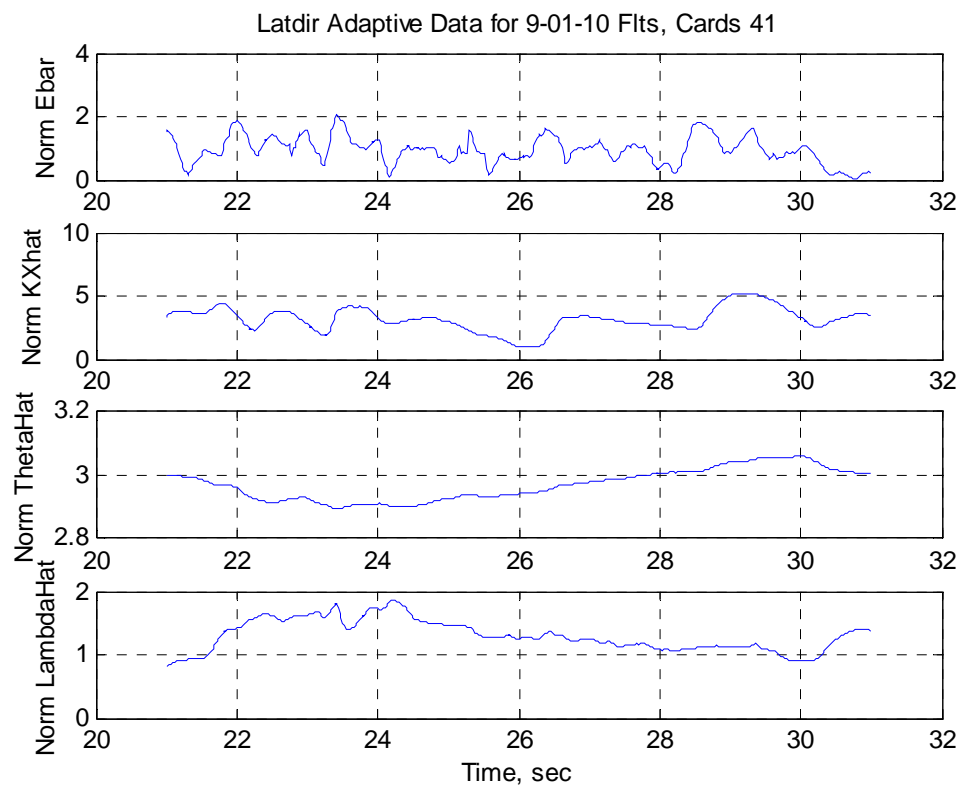

Figure III.45: Lateral/Directional Adaptive Parameters, Research CLAW, Card 41

Finally, the response of the lateral/directional predictor is shown in Figure III.46. The trace indicates that the predictor is working per design. The rudder portion of the predictor shows a slight degradation in performance during the time when the roll rate wavetrain is being injected and tracked.
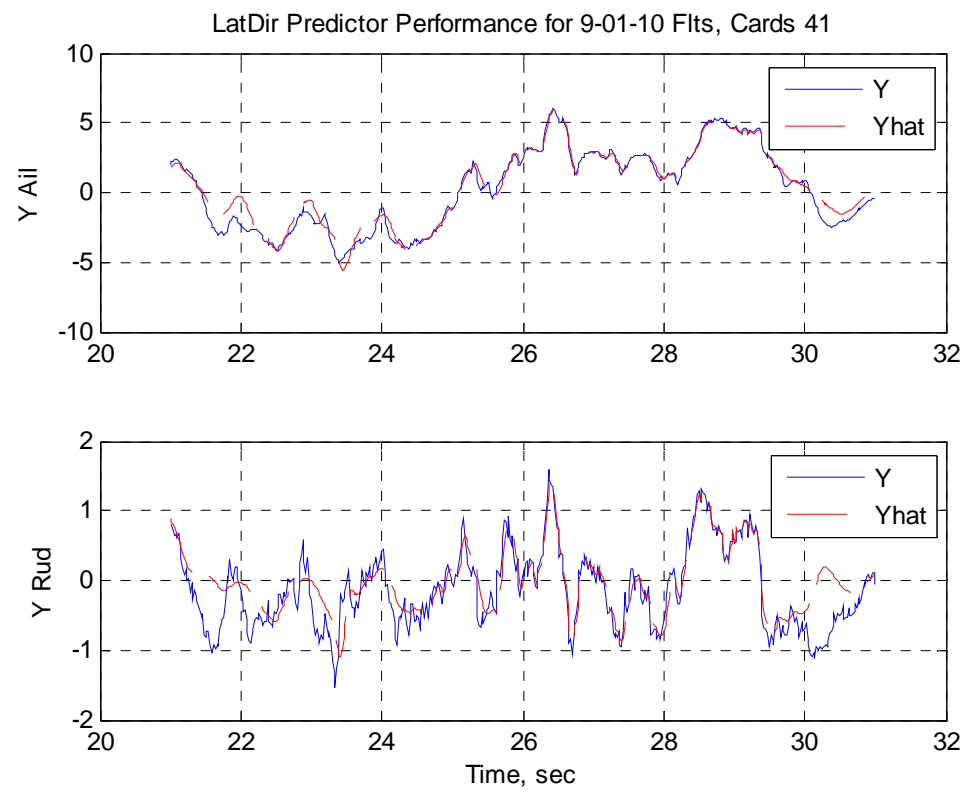

Figure III.46: Lateral/Directional Predictor Performance, Research CLAW, Card 41

For cards 29/43, the aircraft longitudinal response to roll rate wavetrain with $75 \%$ reduction in $C_{m \alpha}$ and $C_{l p}$ is shown in Figure III.47 and Figure III.48. 

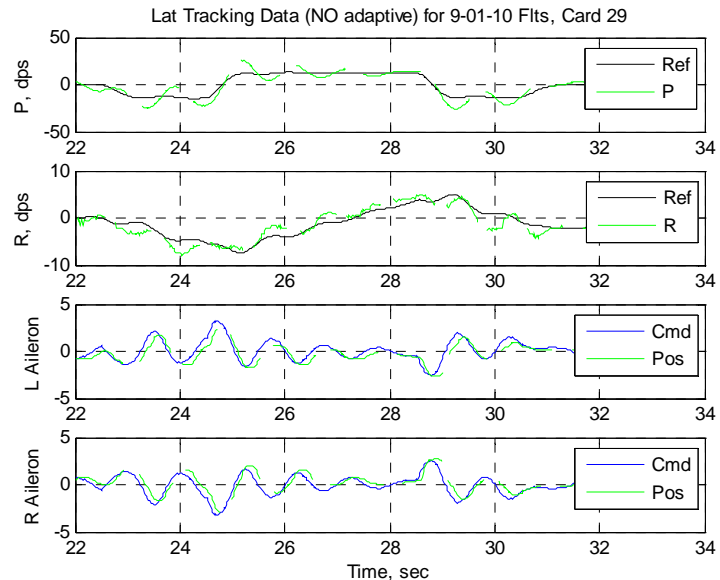

Figure III.47: Lateral Tracking,

Baseline CLAW, Card 29
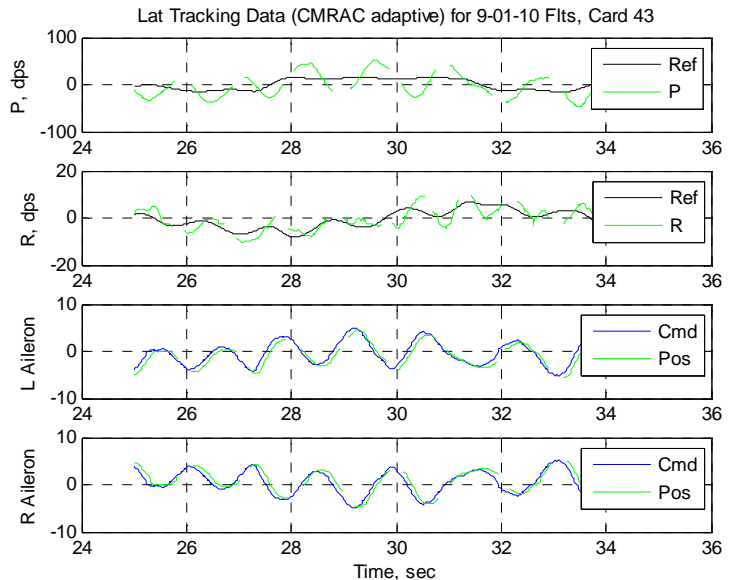

Figure III.48: Lateral Tracking,

Research CLAW, Card 43

The lateral tracking responses show that the baseline control law is able to track the reference model in the presence of degradation with a slightly underdamped response. The research control law appears more oscillatory, with corresponding increase in surface deflection and activity. It is difficult to discern whether effective tracking is being maintained, though the pilot offered no comments for this card and therefore did not appear to be displeased with the research control law response. Once again, the oscillatory response in the research control law may be heavily influenced by the moderate turbulence present for flight 38. The lateral/directional adaptive parameters and predictor performance shown in Figure III.49 and Figure III.50, respectively indicate that the augmentation is working to maintain tracking without objectionable adaptive gains or dynamics.
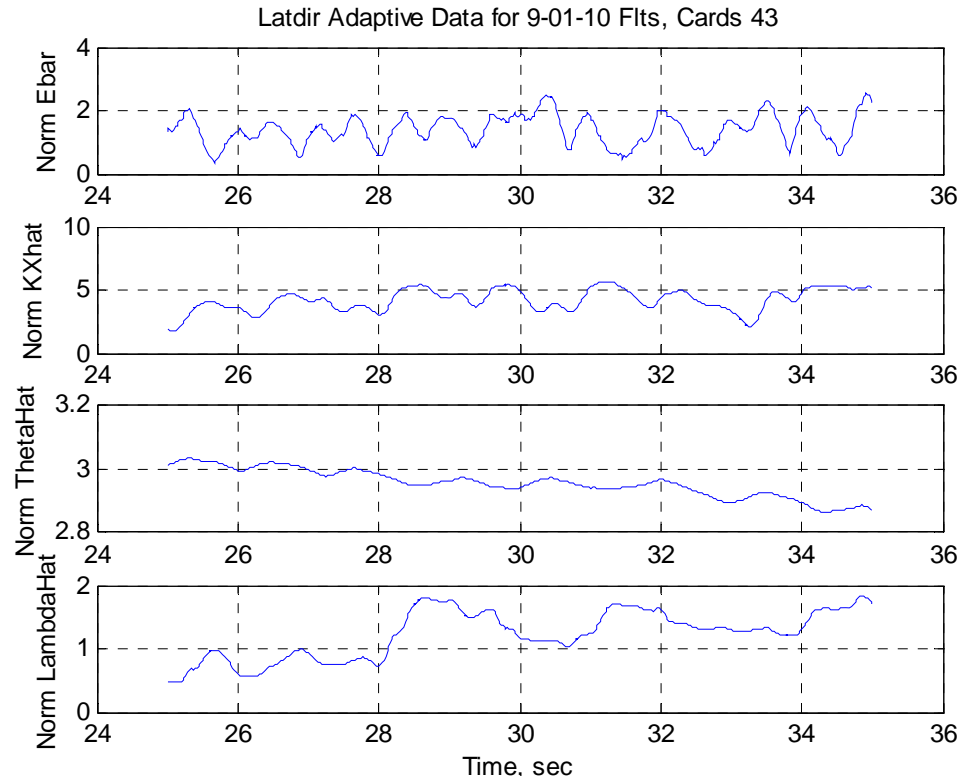

Figure III.49: Lateral/Directional Adaptive Parameters, Research CLAW, Card 43 

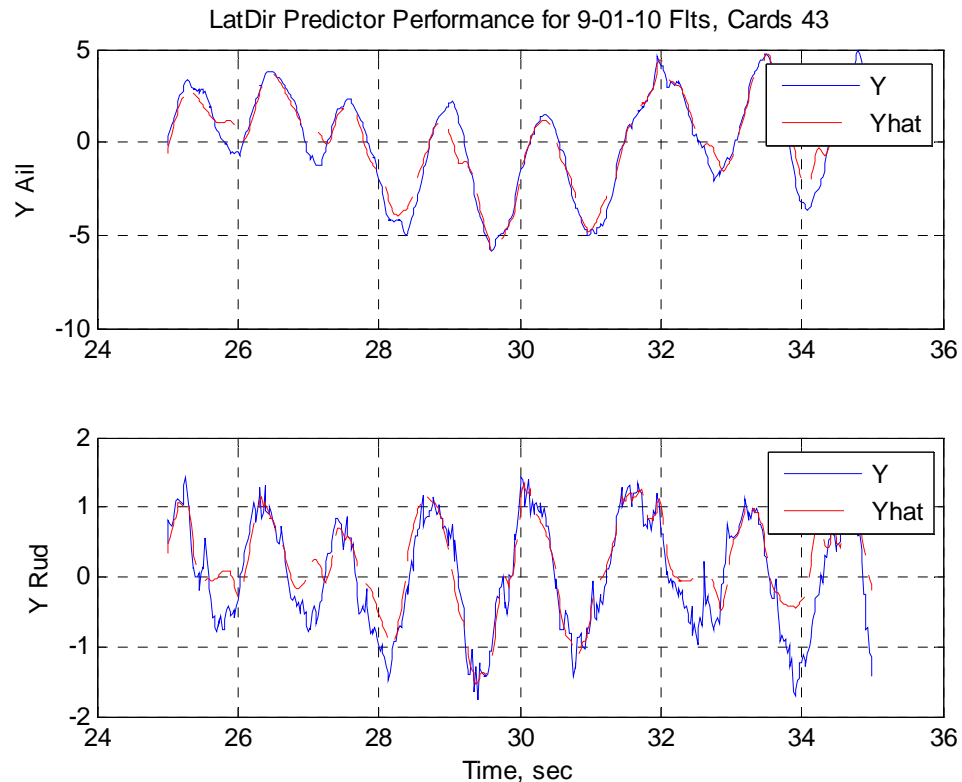

Figure III.50: Lateral/Directional Predictor Performance, Research CLAW, Card 43

The FFT coefficients for this maneuver confirm that the research control law was employing more control effort than the baseline control law, as shown in responses in Figure III.51 and Figure III.52.

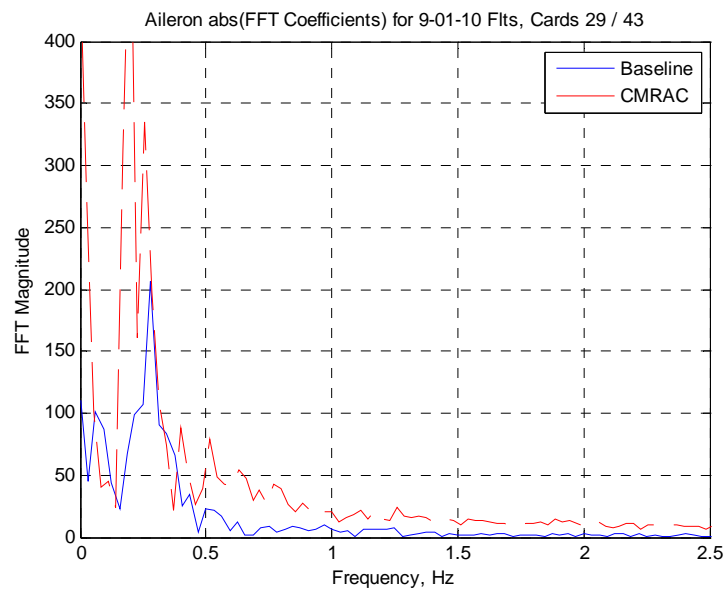

Figure III.51: FFT Coefficients of Aileron

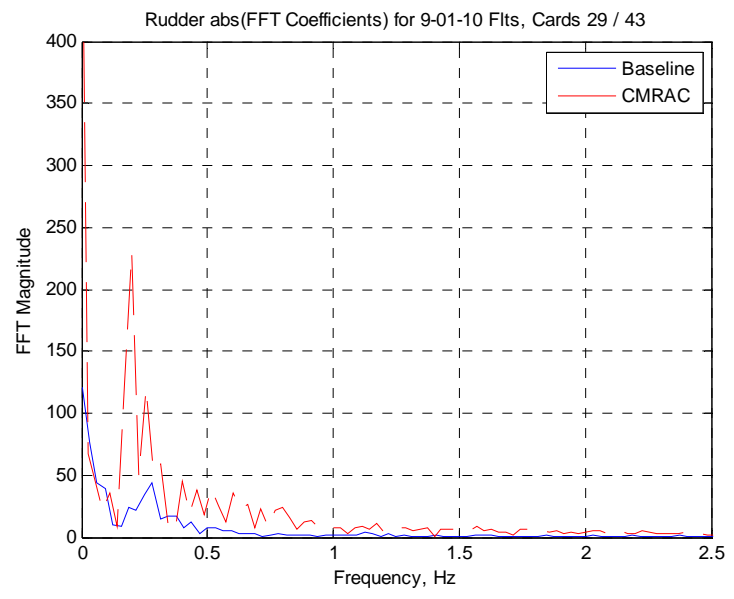

Figure III.52: FFT Coefficients of Rudder

Input, Baseline vs. Research CLAW, Cards 29 / 43

Because of the KIO call for card 44, the roll rate wavetrain with $100 \%$ reduction in $C_{m \alpha}$ and $C_{l p}$ was not attempted for the research control law. The baseline control law was exercised at this level of failure in card 31, and the aircraft lateral and directional responses are shown in Figure III.53 and Figure III.54. 

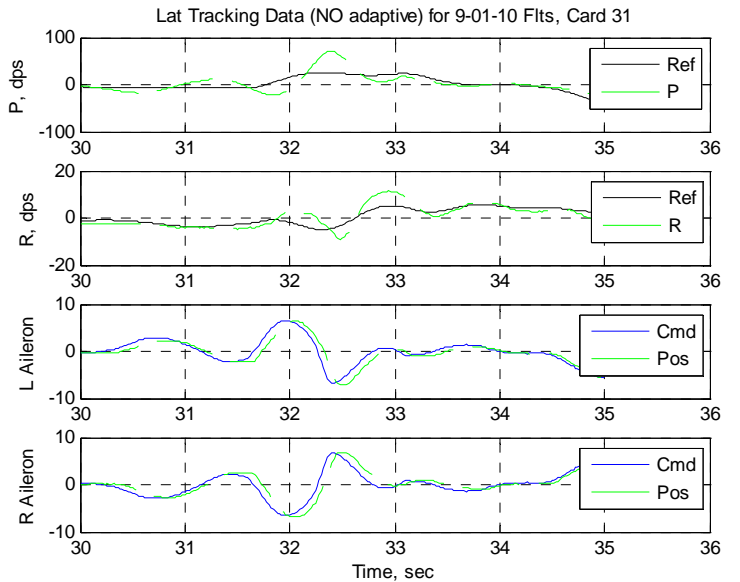

Figure III.53: Lateral Tracking,
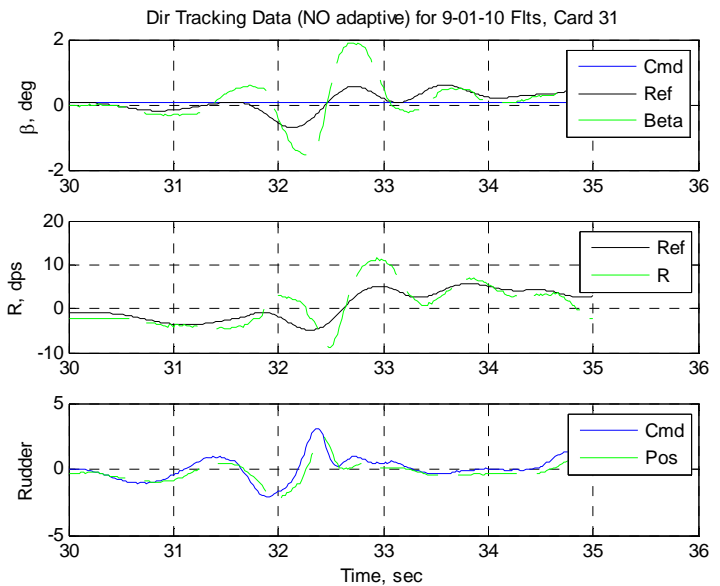

Figure III.54: Directional Tracking,

\section{Baseline CLAW, Card 31}

The responses show a divergent roll rate response soon after injection of the wave train. This matches the pilot comment to KIO shortly after wavetrain engaged.

\section{E. Airborne S\&C Results with Stuck Elevator}

Cards 34/48 injected AOA wavetrain with a fault such that the left elevators are stuck at trim position and only right elevators are available for longitudinal control. The longitudinal response of the vehicle under baseline and research control laws is shown in Figure III.55 and Figure III.56, and shows that only right outboard elevator surfaces is active. In fact, both the inboard and outboard left elevators are stuck at trim value for the maneuver and cannot follow surface commands. The task of AOA tracking is left to the right elevator panels, and for both control laws the AOA response is able to track the reference model closely. Turbulence is once again present in the card 48 response, but the efficacy of the research control law is still displayed.
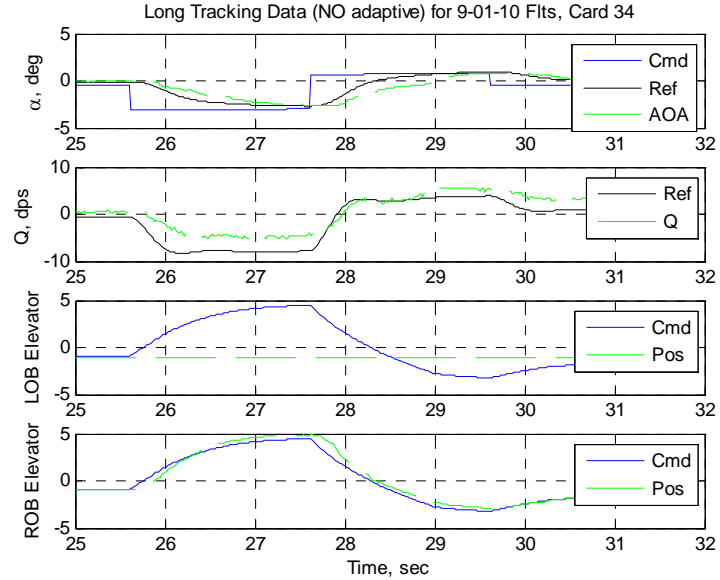

Figure III.55: Longitudinal Tracking, Baseline CLAW, Card 34
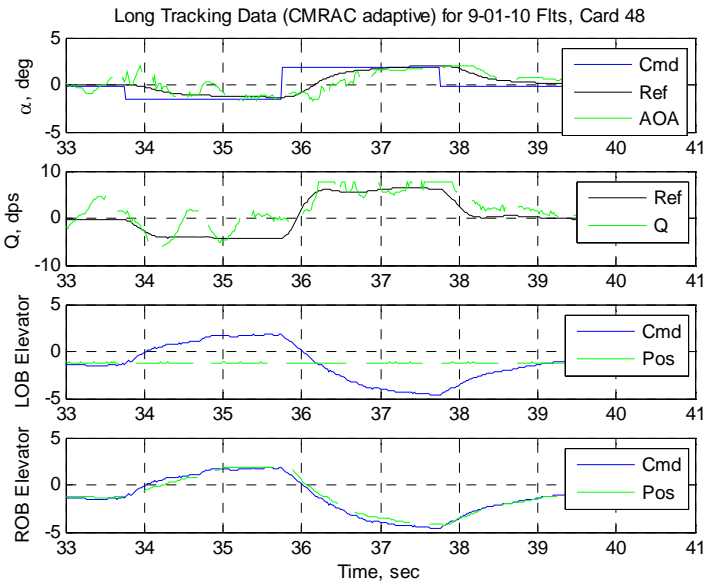

Figure III.56: Longitudinal Tracking, Research CLAW, Card 48

The baseline control system is sufficiently robust to this failure that the adaptive augmentation need provide little input to the system. This is expected, since the baseline LQR+PI architecture should withstand up to $50 \%$ loss of input effectiveness by design. As expected, the longitudinal adaptive parameters show very little activity, as shown in Figure III.57. 

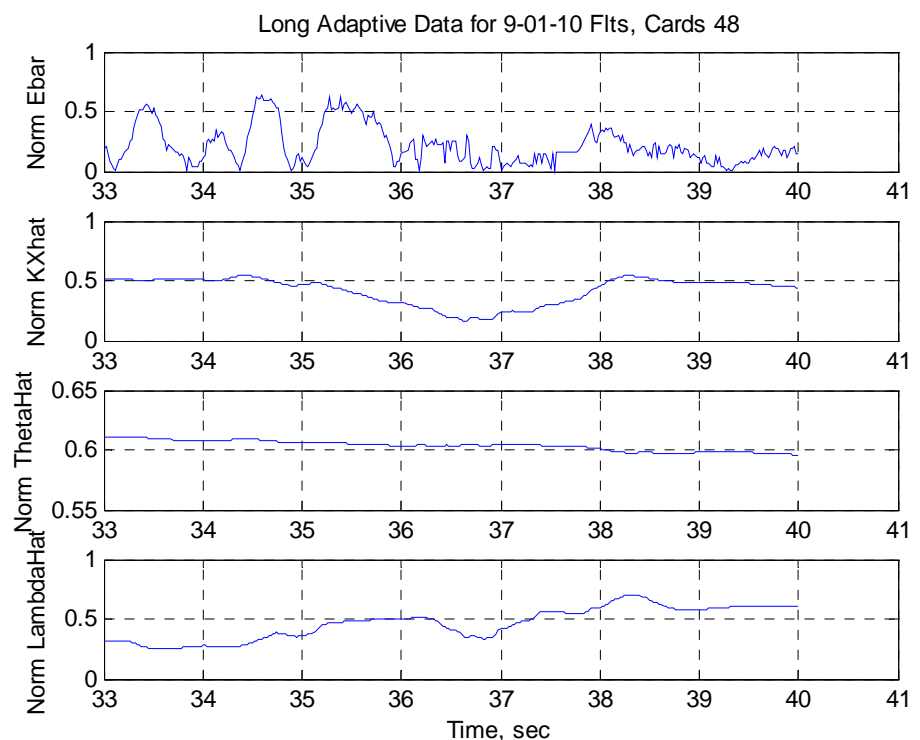

Figure III.57: Longitudinal Adaptive Parameters, Research CLAW, Card 48

\section{F. Flight Test Results - Offset Landing Evaluation}

Flights 42 and 43 were completed on September 10, 2010. The objective of these flights was to perform offset landing evaluation of the research flight control law (Flight 42) and of the baseline flight control law (Flight 43). Just as in the S\&C evaluation, a similar set of flight cards was used for each flight; with the difference being which flight control law was engaged during the associated maneuvers. All cards completed during this flight deployment had a target altitude of 1000 feet, and a target indicated airspeed of between 70 to 85 kts. Notation for the baseline and the research control laws remains consistent with that reported in the S\&C evaluation section.

The first task was to fly each control law without failures present as a practice exercise and to give a nominal Cooper-Harper Rating (CHR) to each control law in the absence of failures. The CHR scale is a method for rating pilot Handling Qualities (HQ) assessments of aircraft response, and is shown in Figure III.58. Since there is no simply defined tracking task (such as the waveform in the S\&C evaluation cases), the CHR gives a measure of the pilot opinion for each control law relevant to his ability to perform the offset landing task. The desired and adequate performance requirements are displayed on the Heads Up Display (HUD) during the task execution.

\section{Cooper-Harper Rating Scale}

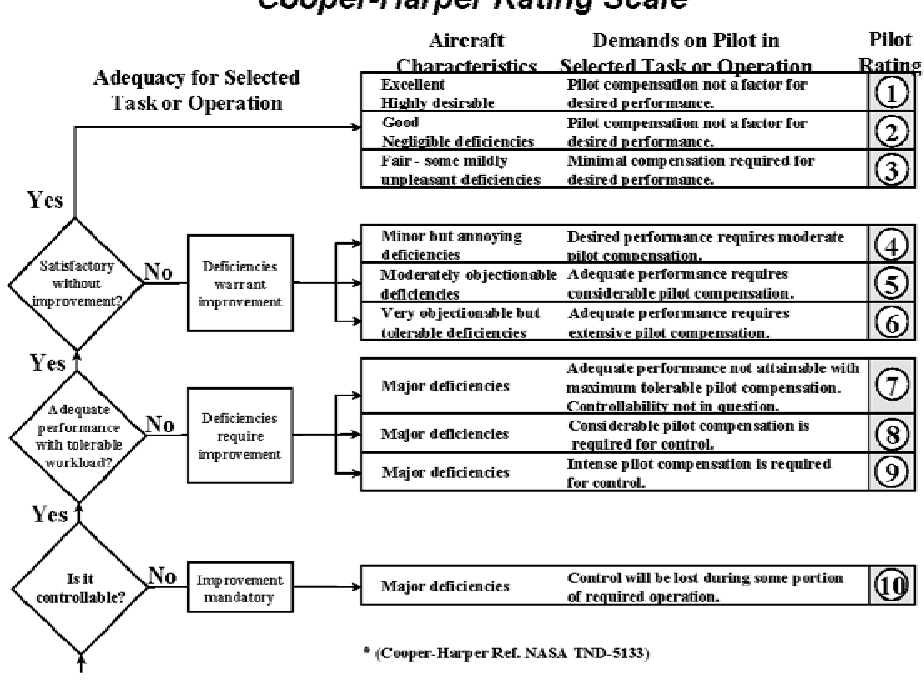

Figure III.58: The Cooper-Harper Rating (CHR) Scale 23

American Institute of Aeronautics and Astronautics 
The particulars of the maneuver executed during flight test are as follows:

- Initial offset : $90 \mathrm{ft}$. lateral, $1800 \mathrm{ft}$. downrange, $100 \mathrm{ft}$. above the runway

- Performance boundaries:

- Desired $-|\phi|= \pm 10$ deg; $|\gamma|= \pm 1$ deg; landing box = 24’x328'

- Adequate $-|\phi|= \pm 20 \mathrm{deg} ;|\gamma|= \pm 3$ deg; landing box = 9’x30'

- Flying qualities ratings taken for nominal, neutrally stable, and unstable airplane

The lateral/directional results of the offset landing task in the absence of failure for research control law and research control law in cards 83/84 are shown in Figure III.59 and Figure III.60.
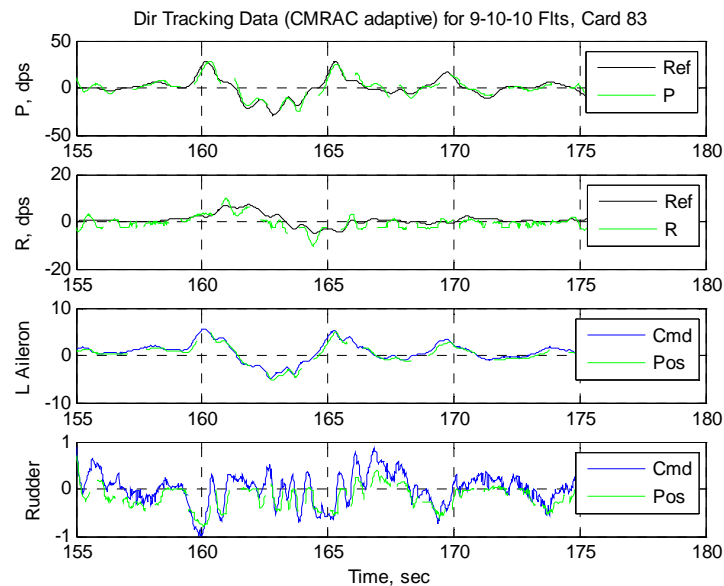

Figure III.59: Lateral / Directional Data, Research CLAW, Card 83
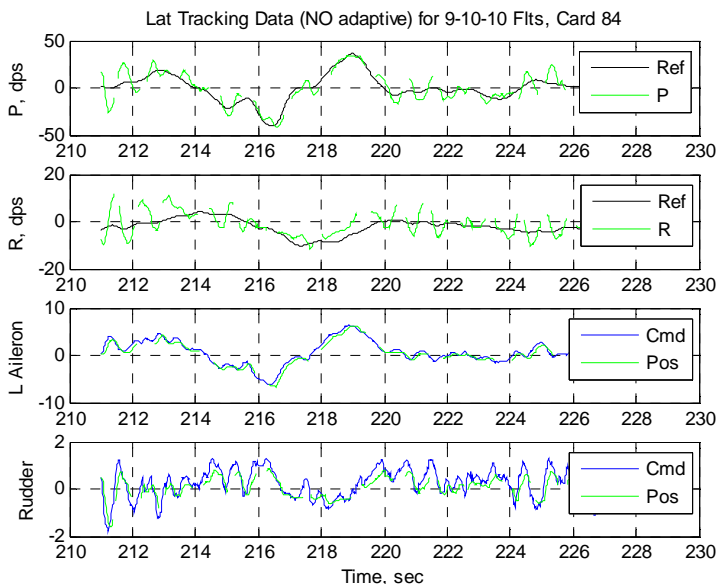

Figure III.60: Lateral/Directional Data, Baseline CLAW, Card 84

The results show that both control laws are able to track the reference dynamics, with the baseline control law showing more oscillatory response in the yaw channel. This is at least partially attributable to turbulence, as the baseline control law offset landing flight experienced significant turbulence. Even in a challenging offset landing maneuver, the lateral/directional adaptive parameters shown in Figure III.61 are well-behaved and do not contain undesirable magnitude nor dynamics.
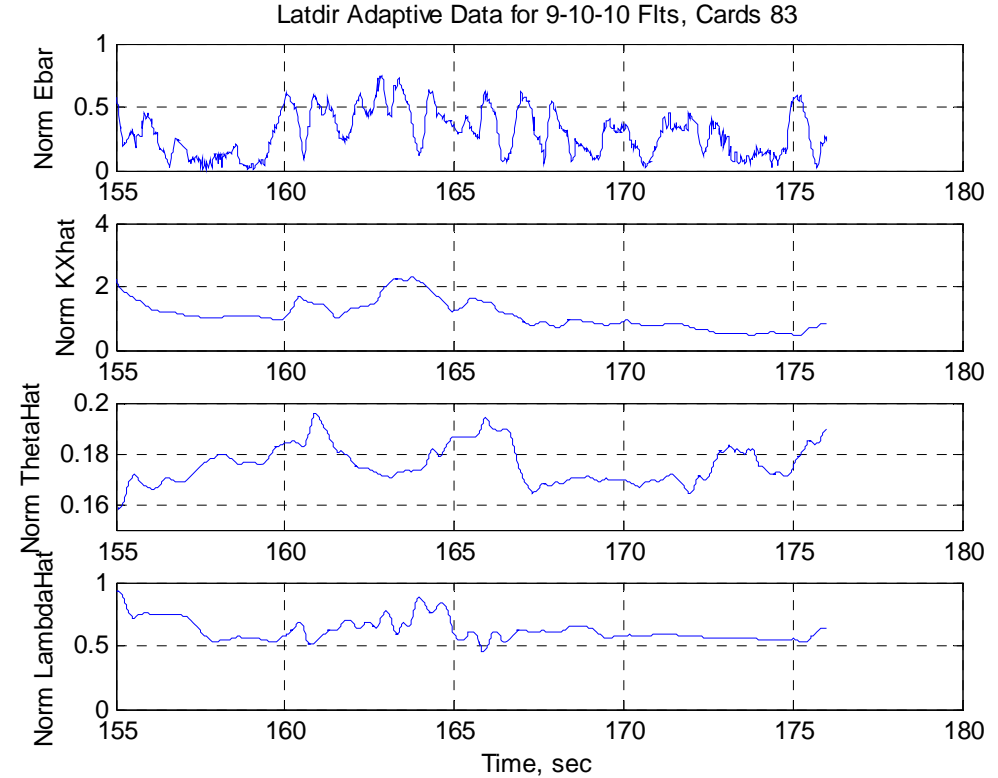

Figure III.61: Lateral/Directional Adaptive Parameters, Research CLAW, Card 83

24

American Institute of Aeronautics and Astronautics 
In addition the lateral/directional predictor is operating per design and is adequately predicting the system dynamics, albeit with some mismatch due to the turbulence / external disturbance acting on the system, as shown in Figure III.62.
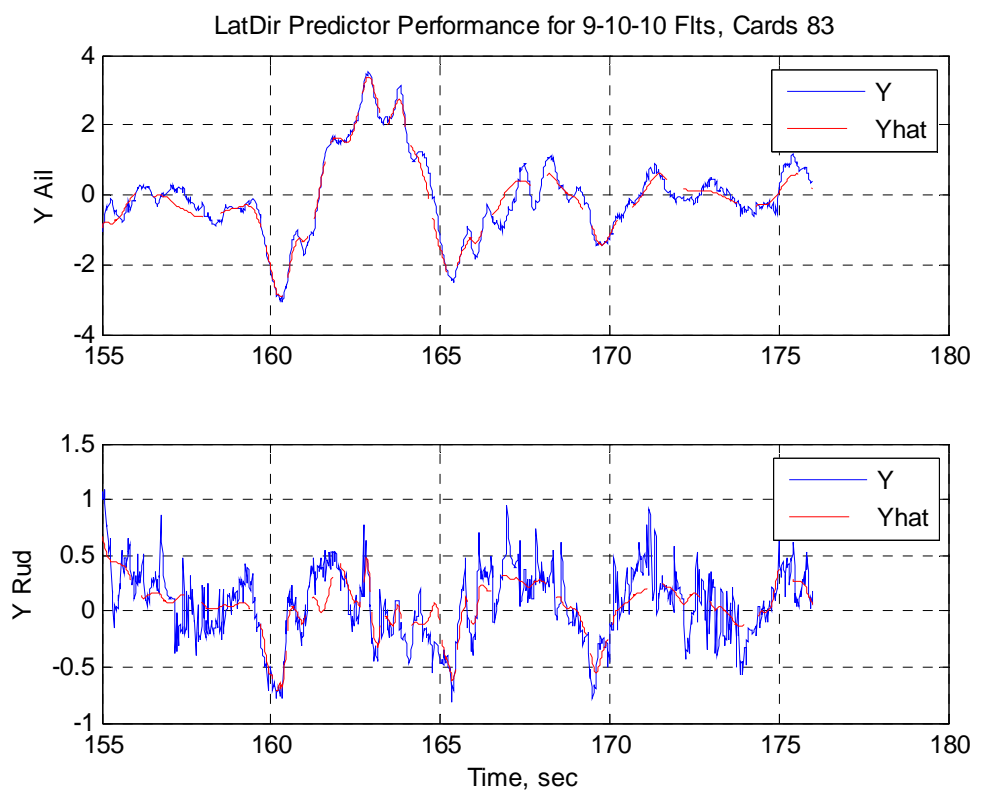

Figure III.62: Lateral/Directional Predictor Performance, Research CLAW, Card 83

Finally, comparisons of the FFT coefficients of the aileron and rudder surfaces show that the surface activity used by each control law is comparable. The data are shown in Figure III.63 and Figure III.64.

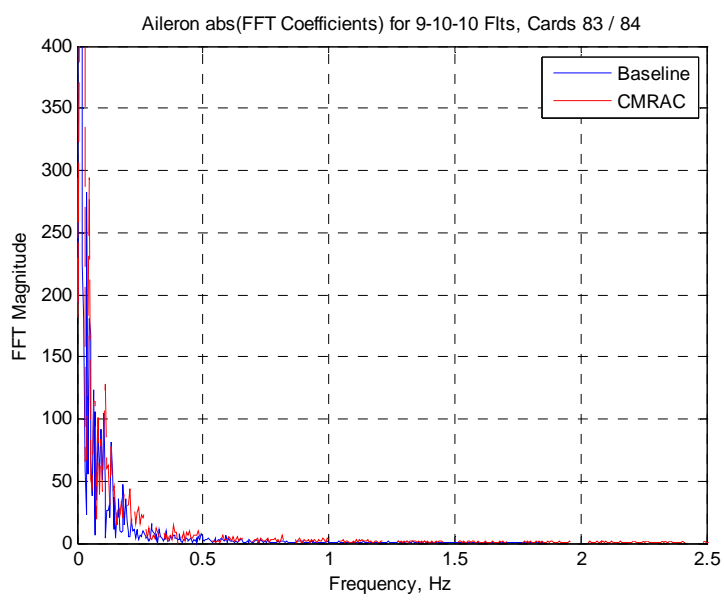

Figure III.63: FFT Coefficients of Aileron

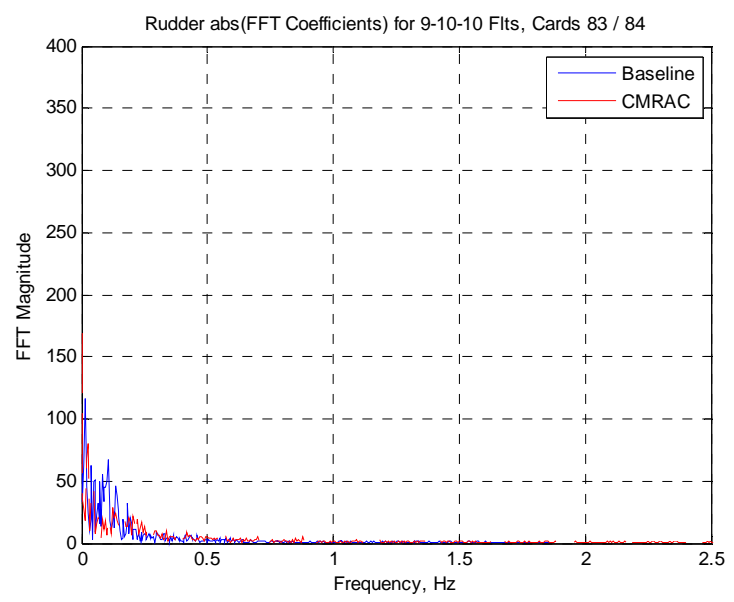

Figure III.64: FFT Coefficients of Rudder

Input, Baseline vs. Research CLAW, Cards 83/84

In summary, the reviewed offset landing cases (absence of failures) indicate that the research control law was able to provide similar tracking performance and comparable surface activity to the baseline control law. Simultaneously, the research control law maintained acceptable adaptive parameter activity. Both control laws received CHR 4 from the pilot, with the comment that in the baseline control law flight it "looks like turbulence is exciting the lat/dir axes." The pilot noted that he took turbulence into consideration when providing the CHR rating.

The next set of cards was a repeat of cards 83/84, this time with $100 \%$ reduction in $C_{m \alpha}$ and $C_{l p}$. These modifications of the aircraft aerodynamics are achieved in the same fashion as for the airborne S\&C evaluation 
flights. The lateral/directional responses of the research and baseline control law are shown in Figure III.65 and Figure III.66 respectively. The results indicate that the baseline control law had larger roll oscillations and aileron magnitude than the research control law, with similar rudder magnitude. It is not clear how much the turbulence may have contributed to this response. The research control law received CHR 4 from the pilot, while the baseline control law received CHR 7.

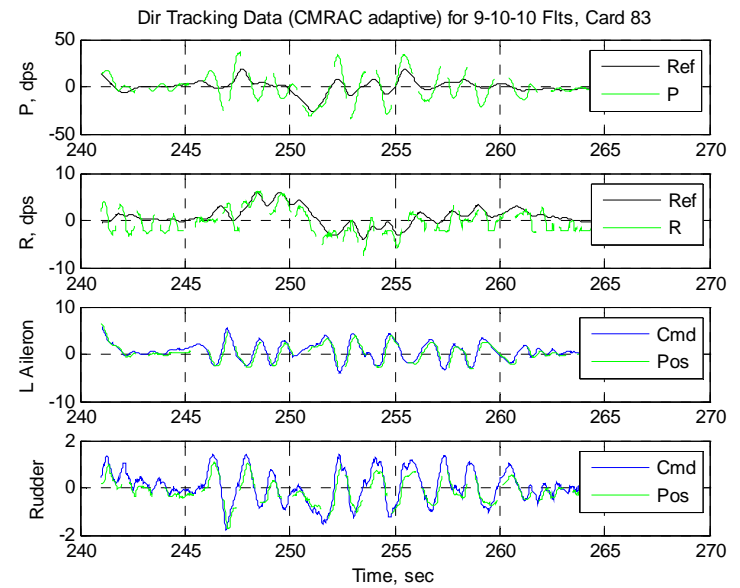

Figure III.65: Lateral/Directional Data, Research CLAW, Card 83
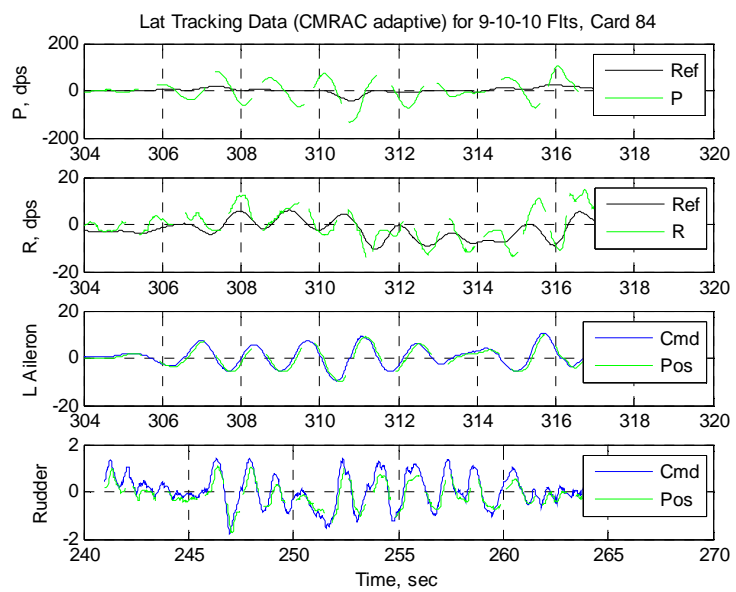

Figure III.66: Lateral/Directional Data,

Baseline CLAW, Card 84

The lateral/directional adaptive parameters shown in Figure III.67 indicate that at the beginning of the offset landing maneuver, some adaptive variables noticeably increased in magnitude. This change may have been a contributor to the oscillatory response for the research control law, as the overall control gain may have been high. It should be noted that the reference models and adaptive weights employed by the research control law were the same for all flight conditions. In other words, the design was done for a single point condition in the airborne flight regime and was not adjusted nor scheduled for tasks such as offset landing. This may have also contributed to the response of the research control law, as the lateral/directional axes may require additional adjustment and analysis prior to application throughout the flight envelope and under high gain control tasks such as offset landing.

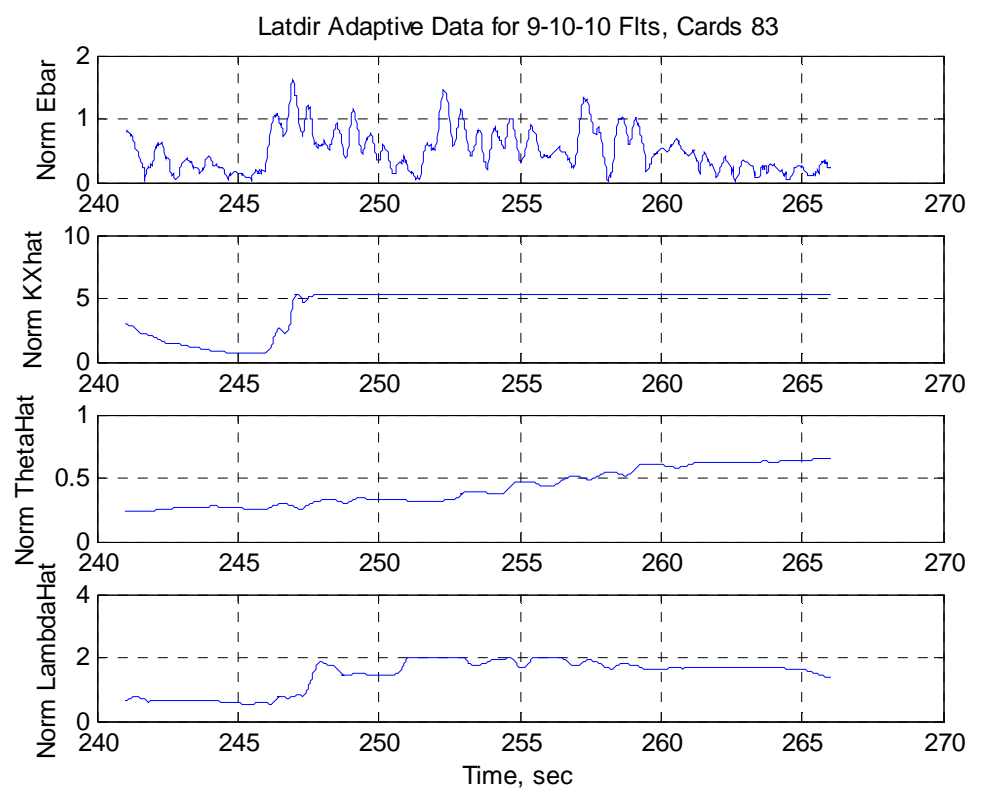

Figure III.67: Lateral/Directional Adaptive Parameters, Research CLAW, Card 83 
While the research control law does have an oscillatory response and may need adjusting, it should be noted that the predictor portion of CMRAC still functioned per design and did an acceptable job of one-step-ahead estimation, as shown in Figure III.68.
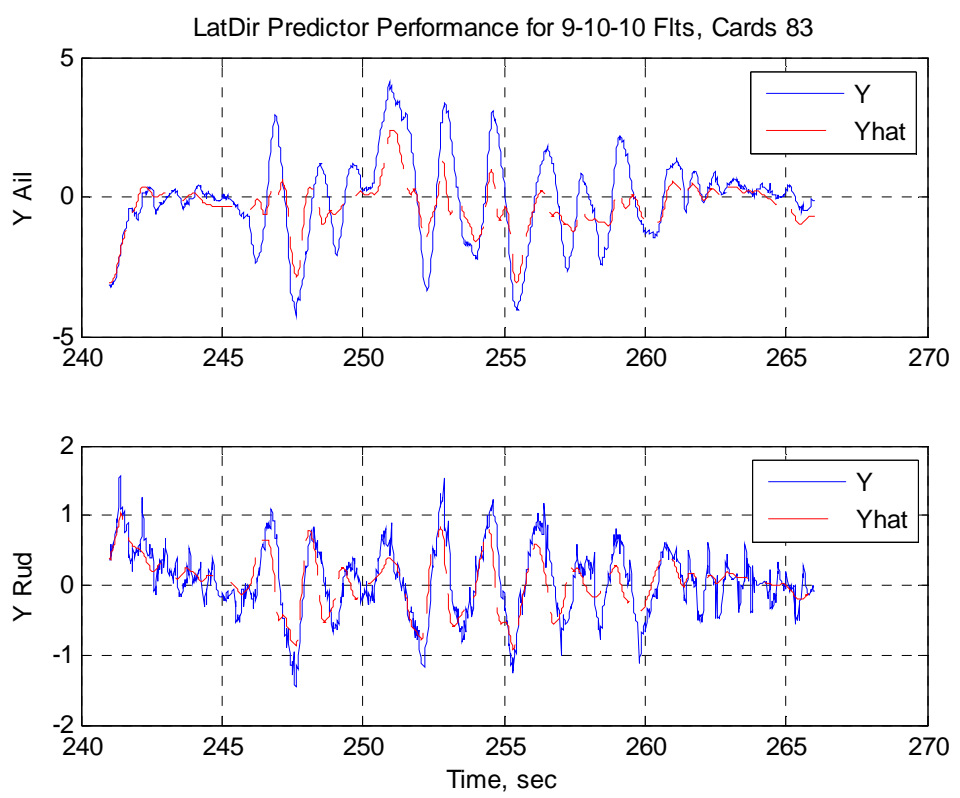

Figure III.68: Lateral/Directional Predictor Performance, Research CLAW, Card 83

The effectiveness of the CMRAC architecture can also be seen in the plots of the FFT coefficients of the aileron and rudder surfaces, which are shown in Figure III.69 and

Figure III.70 respectively. Once again, the research control law uses similar surface activity, even in the presence of an oscillatory response while providing siginificantly better handling qualities than the baseline.

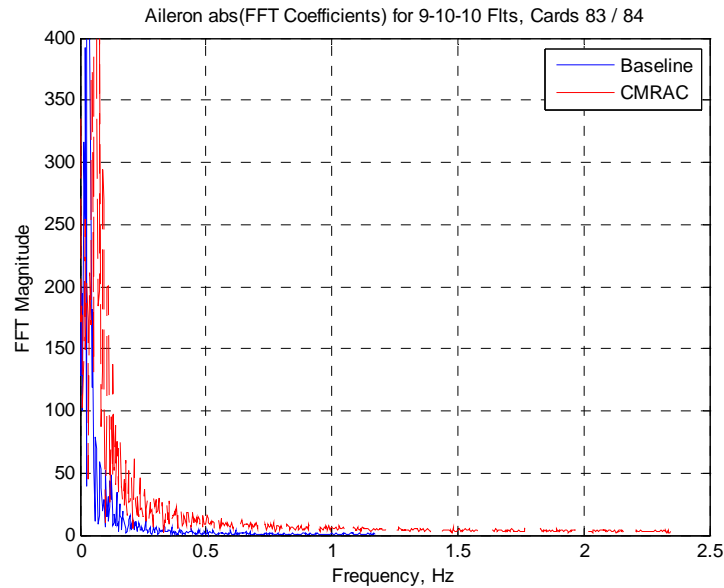

Figure III.69: Coefficients of Aileron

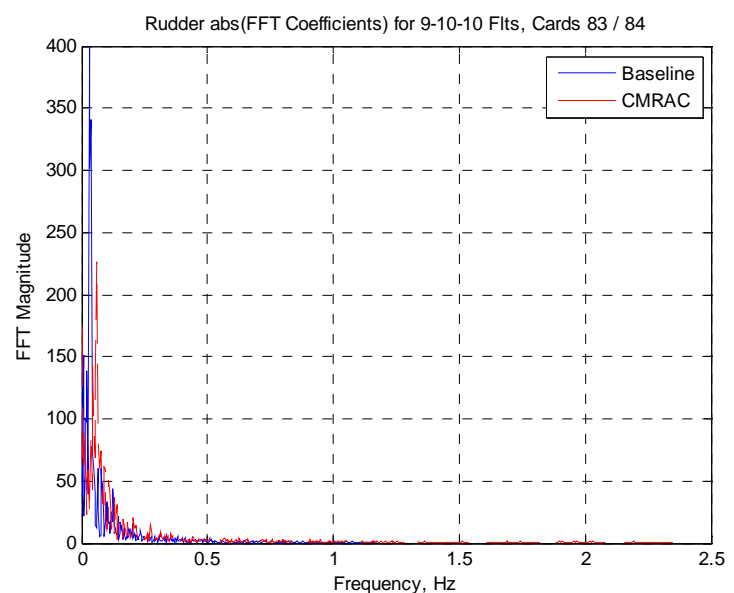

Figure III.70: Coefficients of Rudder

Input, Baseline vs. Research CLAW, Cards 83/84

The final set of cards was a repeat of cards 93/94, this time with $125 \%$ reduction in $C_{m \alpha}$ and $C_{l p}$, unstable airplane. The lateral/directional responses of the research and baseline control law are in Figure III.71 and Figure III.72 respectively. The baseline control law shows roll rate oscillations well in excess of 100 degrees/second, with the pilot giving CHR 9 and stating that the turbulence did not make a difference in this case. Meanwhile the research control law also generated significant roll rates and was described by the pilot as "a wild ride" and rated CHR 7. 

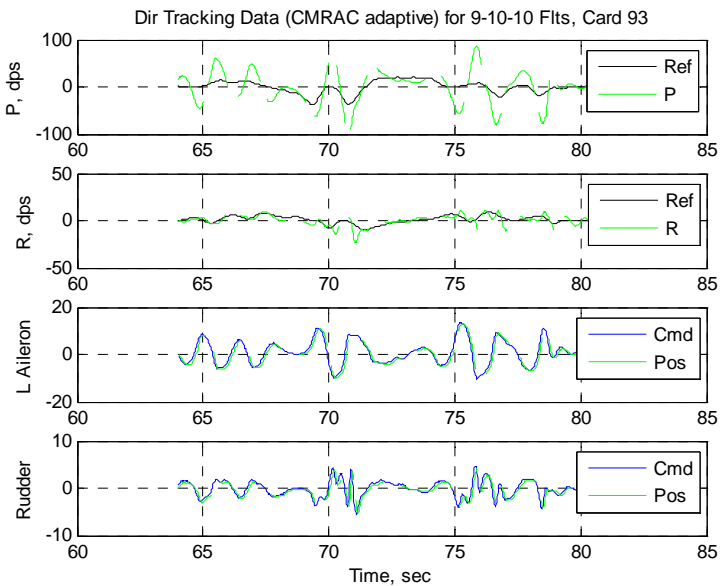

Figure III.71: Lateral/Directional Data, Research CLAW, Card 93
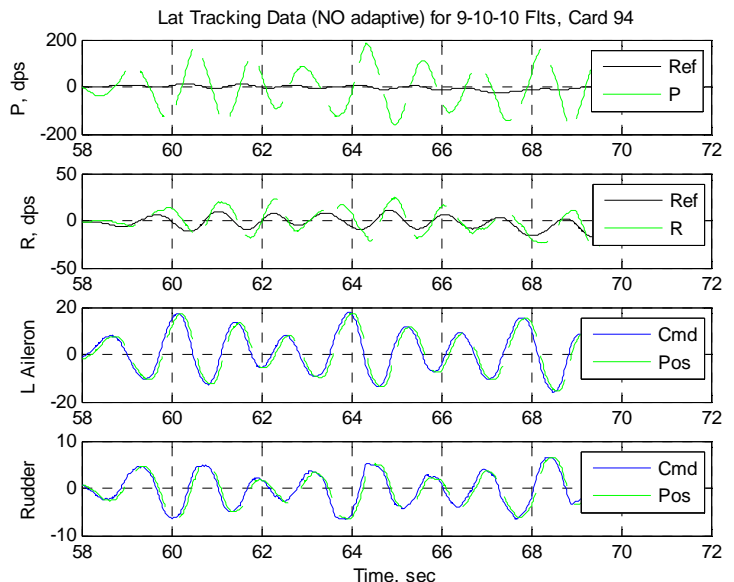

Figure III.72: Lateral/Directional Data, Baseline CLAW, Card 94

As mentioned in the previous card set, it is possible that the research CLAW needed adjustment, because as the lateral/directional adaptive parameters show in Figure III.73, the KXhat adaptive parameter is hitting its projection bound throughout the maneuver. This indicates that adaptive augmentation may be contributing too much gain and that the reference model and/or learning rates may need adjustment.
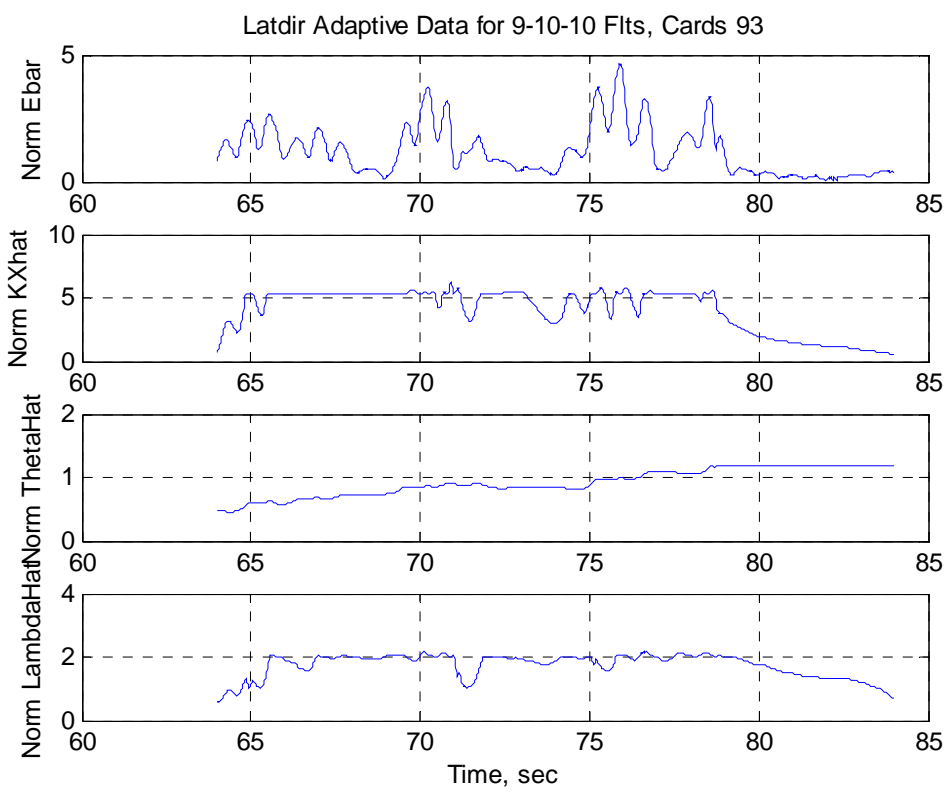

Figure III.73: Lateral/Directional Adaptive Parameters, Research CLAW, Card 93

Similar to results from the previous card, although the adaptive parameters are more active than desired and potentially causing high gain, the predictor portion of CMRAC architecture is showing desirable response and estimating system dynamics, as shown in Figure III.74. 

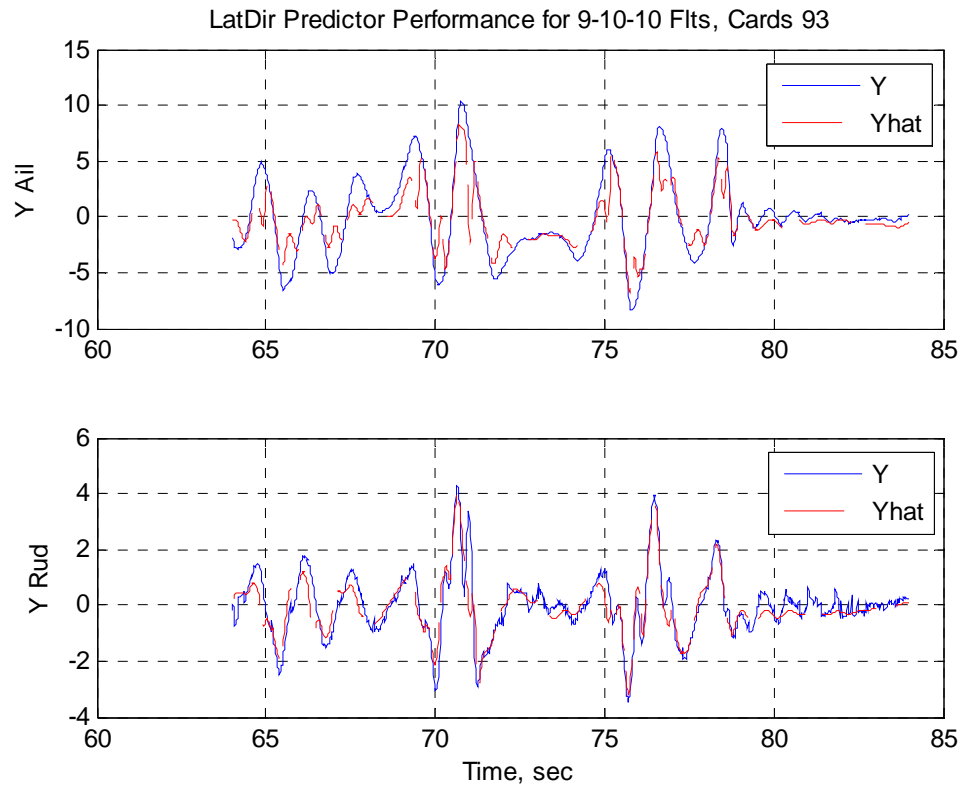

Figure III.74: Lateral/Directional Predictor Performance, Research CLAW, Card 93

Finally, the plots of the FFT coefficients of the aileron and rudder control signals shown in Figure III.75 and Figure III.76 respectively indicate that once again, despite oscillatory response and turbulence, the CMRAC research control law produces reasonable level of control input while producing better HQ. This is one of the design benefits of the CMRAC architecture compared to classical MRAC.

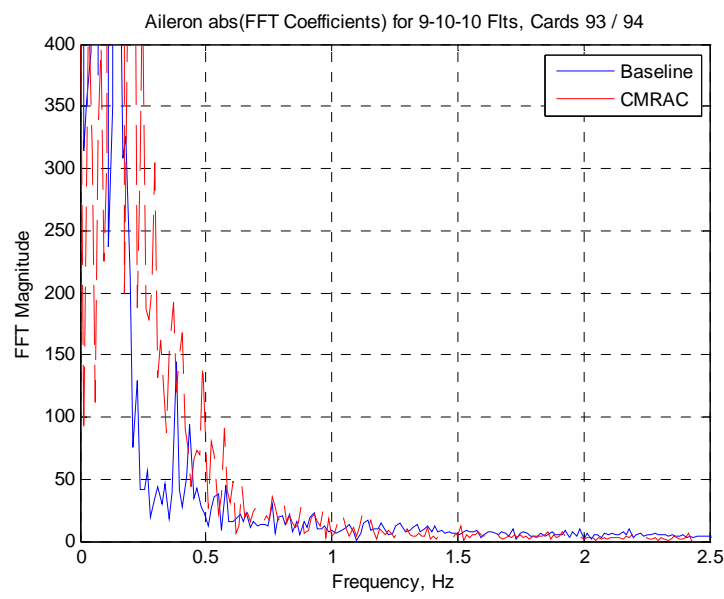

Figure III.75: Coefficients of Aileron

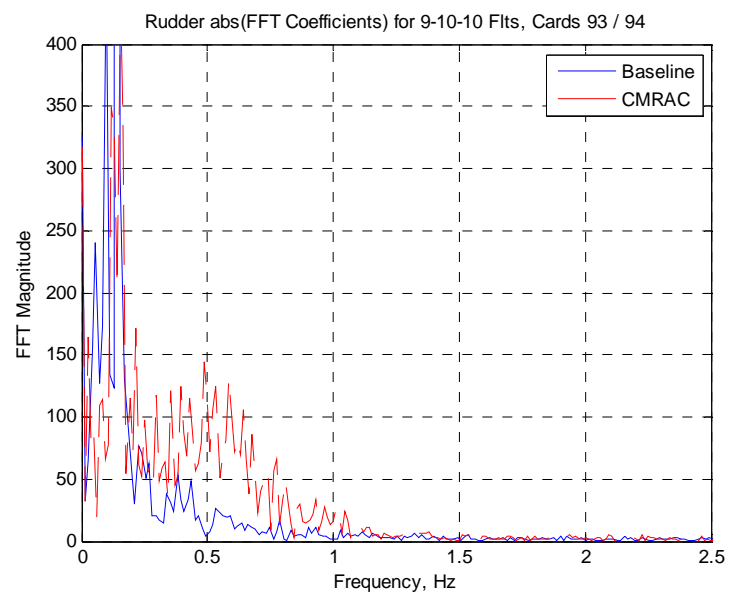

Figure III.76: Coefficients of Rudder

Input, Baseline vs. Research CLAW, Cards 93/94

\section{Conclusion}

This paper presents the results of the September 2010 flight test deployment of the NASA AirSTAR dynamically scaled twin turbine-powered generic Transport Model aircraft utilizing Boeing-designed control laws. The baseline LQR+PI and the research CMRAC control laws were evaluated in both airborne S\&C wavetrain tracking tasks, as well as during simulated offset landing tasks, with various levels of failures.

The baseline control law design was shown to meet tracking requirements based on the pre-selected reference model. This controller was also shown to be robust to a variety of failures including time-delays, control 
effectiveness reduction and stuck surfaces. The research control law similarly showed acceptable level of tracking performance and robustness. It is difficult to draw concrete comparisons between the two control schemes due to the somewhat varying nature of the flight test maneuvers, and because the level of turbulence varied slightly in the back-to-back flights that evaluated the baseline and the research control laws. However, some overall comments can be made. The research control law in general showed graceful performance degradation in the presence of reduced control effectiveness and comparable robustness to time delays. At the higher levels of aircraft destabilization, the research control law led to more oscillatory response.

As the theory suggests, the CMRAC architecture is able to restore tracking in the presence of failures, while simultaneously using reduced control surface activity to meet the tracking goals. This was seen in flight test results even in cases where the research control law had oscillatory response. The reference model was only designed for a single point flight condition and maneuver set without taking into account failures and latencies used in flight, nor taking into account high gain tasks such as offset landing. As such, the flight test results serve to validate the effectiveness of the CMRAC architecture in general with the understanding that for a practical application and deployment, traditional full-envelope analyses and possibly reference model scheduling should be performed.

\section{Acknowledgments}

The authors would like to thank the staff of the AirSTAR Flight Test Facility for their support with control law implementation, their insights into flight dynamics and piloted evaluations. This work was sponsored by NASA grant number NNL06AA04B. The views and conclusions contained herein are those of the authors and should not necessarily be interpreted as representing the official policies or endorsements, either expressed or implied, of NASA or the U.S. Government.

\section{References}

[1] Murch, A. M., Cox, D. E., and Cunningham, K., "Software Considerations for Subscale Experimental Control Laws," Infotech@Aerospace Conference, AIAA Paper 2009-2054, Seattle, WA, 2009.

[2] Cunningham, K., Foster, J. V., Morelli, E. A., and Murch, A. M., "Practical Application of a Subscale Transport Aircraft for Flight Research in Control Upset and Failure Conditions,” Atmospheric Flight Mechanics Conference, AIAA Paper 20086200, Honolulu, HI, 2008.

[3] Murch, A. M., “A Flight Control System Architecture for the NASA AirSTAR Flight Test Infrastructure,” Guidance, Navigation, and Control Conference, AIAA Paper 2008-6990, Honolulu, HI, 2008.

[4] Jordan, T. L., Foster, J. V., Bailey, R. M., and Belcastro, C. M., "AirSTAR: A UAV Platform for Flight Dynamics and Control System Testing,” Aerodynamic Measurement Technology and Ground Testing Conference, AIAA Paper 20063307, San Francisco, CA, 2006.

[5] Jordan, T. L., Langford, W. M., and Hill, J. S., “Airborne Subscale Transport Aircraft Research Testbed - Aircraft Model Development,” Guidance, Navigation, and Control Conference, AIAA Paper 2005-6432, San Francisco, CA, 2005.

[6] Bailey, R. M., Hostetler, R. W., Barnes, K. N., Belcastro, Christine M., and Belcastro, Celeste M., “Experimental Validation: Subscale Aircraft Ground Facilities and Integrated Test Capability,” Guidance, Navigation, and Control Conference, AIAA Paper 2005-6433, San Francisco, CA, 2005.

[7] Narendra, K. S., and Annaswamy, A. M., Stable Adaptive Systems, Dover, 2005.

[8] Ioannou, P. A., and Fidan, B., Adaptive Control Tutorial, SIAM, Philadelphia, 2006.

[9] Slotine, J.-J. E., and Li, W., Applied Nonlinear Control, Prentice Hall, 1995.

[10] Lavretsky, E., “Combined/Composite Model Reference Adaptive Control,” IEEE Transactions on Automatic Control, 54(11), pp. 2692-2697, 2009.

[11] Gadient, R., Levin, J., and Lavretsky, E., "Comparison of Model Reference Adaptive Controller Designs Applied to the NASA Generic Transport Model,” Guidance, Navigation, and Control Conference, AIAA Paper 2010-8406, Toronto, ON, 2010.

[12] Cunningham, K. “AirSTAR Flight Test Plan: 5.5 \% Dynamically Scaled GTM Tail Number T2. Deployment: 2010.02.” GTMP-6326 2010.02, V 2.00, May 24, 2010.

[13] Cunningham, K. “AirSTAR Flight Test Cards, v2.10.” GTMP-6325 2010.02, V 2.10, May 27, 2010. 